\title{
TUTTE POLYNOMIALS OF TENSOR PRODUCTS OF SIGNED GRAPHS AND THEIR APPLICATIONS IN KNOT THEORY
}

\author{
Y. DIAO, G. HETYEI AND K. HINSON
}

\begin{abstract}
It is well-known that the Jones polynomial of an alternating knot is closely related to the Tutte polynomial of a special graph obtained from a regular projection of the knot. Relying on the results of Bollobás and Riordan, we introduce a generalization of Kauffman's Tutte polynomial of signed graphs for which describing the effect of taking a signed tensor product of signed graphs is very simple. We show that this Tutte polynomial of a signed tensor product of signed graphs may be expressed in terms of the Tutte polynomials of the original signed graphs by using a simple substitution rule. Our result enables us to compute the Jones polynomials of some large non-alternating knots. The combinatorics used to prove our main result is similar to Tutte's original way of counting "activities" and specializes to a new, perhaps simpler proof of the known formulas for the ordinary Tutte polynomial of the tensor product of unsigned graphs or matroids.
\end{abstract}

\section{INTRODUCTION}

It is well-known that the Jones polynomial of a knot is related to the Tutte polynomial of a special graph obtained from a regular projection of the knot [7, 8, 9. It should be noted however, that Tutte's original polynomial [12] is most easily related to an alternating knot. If we want to compute the Jones polynomial of a non-alternating knot, we need to use a signed generalization of the Tutte polynomial, such as the one introduced by Kauffman [9]. Tutte defined his polynomial 12 in terms of counting activities with respect to a specific labelling of the graph, and his main result is showing that the polynomial he introduced is independent of the labelling, thus truly an invariant of the graph. This definition and result may be easily generalized to matroids. The greatest challenge in generalizing Tutte's polynomial to signed graphs or matroids is to preserve the independence of the labelling. This challenge is typically met by considering the Tutte polynomial of a signed graph as an element of a polynomial ring modulo certain relations between the variables. The most general result in the area is due to Bollobás and Riordan [1] who give a necessary and sufficient set of relations modulo which a Tutte polynomial of a signed graph is labelling independent. In particular, Kauffman's Tutte polynomial for signed graphs is a homomorphic image of the most general Tutte polynomial introduced by Bollobás and Riordan.

Our main result consists of generalizing the known formulas for the Tutte polynomial of the tensor product of two matroids to signed graphs and matroids. The tensor product of matroids was introduced by Brylawski [3] who also expressed the Tutte polynomial of a tensor product in terms of the Tutte polynomials of the original graphs [4]. This result allowed Jaeger, Vertigan and Welsh [7] to express the Jones polynomials of some alternating knots. Our signed generalization allows us to compute the Jones polynomials of some similarly complex knots that do not need to be alternating.

1991 Mathematics Subject Classification. 57M25.

Key words and phrases. knots, Jones polynomials, Tutte polynomials, signed graphs, tensor product of graphs. 
In the Preliminaries we review the Bollobás-Riordan definition of a signed Tutte polynomial, using a notation that is closer to Kauffman's paper [9. We introduce a signed Tutte polynomial in a factor ring that is a proper homomorphic image of the most general signed Tutte polynomial but, using a corollary from [1] and a result from the theory of determinantal rings, we observe that the signed Tutte polynomial we propose is a most general possible, if we consider only factor rings that are integral domains. In particular, our proposed signed Tutte polynomial still allows us to compute Kauffman's signed Tutte polynomial and later the Jones polynomial of an associated knot.

We introduce our definition of a signed tensor product of signed graphs in Section 3, Since our aim is to apply this notion to graphs associated to knots, our terminology implies considering connected graphs only. However, our definitions, statements and proofs may be generalized without any substantial change to signed matroids, very much by the same reasons as the ones observed in [1, Remark $3]$.

In Section 4 we generalize two polynomials associated to a graph with a distinguished edge, used by Jaeger, Vertigan and Welsh [7] in their formula for the unsigned tensor product. These polynomials are defined by a system of equations in [7, we define their generalizations in terms of (labelling dependent) "activities" in the spirit of Tutte's paper [12, and then show that our polynomials satisfy a system of equations generalizing the defining equations of [7]. The fact that these polynomials we introduce are labelling independent follows from the equations having a unique solution in an integral domain. This section is the reason why we work with a most general integral domain but not the most general ring in which a Tutte polynomial of signed graphs may be defined.

Our main result is in Section [5. We provide an explicit substitution rule expressing the Tutte polynomial of a signed tensor product of signed graphs in terms of the Tutte polynomials of the original graphs. After showing that the proposed substitution induces an endomorphism of the ring to which our Tutte polynomials belong, it is sufficient to verify our rule for one specific set of representatives, associated to one specific labelling of the edges. The proof then becomes a classic enumeration of "activities" the way, we believe, Tutte would have proved this result. Our proof may also be applied to unsigned graphs, and is perhaps more accessible to a non-expert than the known proofs of the tensor product formulas for unsigned graphs and matroids.

Our study is partly motivated by applications of knot theory to physical and biological sciences in recent years, where there is a need to distinguish different knots and measure the complexity of knots. One common measure of knot complexity is the crossing number of a knot, which is defined as the minimum number of crossings over all possible regular projections of all possible space realizations of the given knot. A remarkable result derived from the Jones polynomial states that if a knot has a reduced alternating projection (i.e. the knot is an alternating knot), then the crossing number of the knot is equal to the number of crossings in this reduced alternating projection [8, 10, 11. Furthermore, for non-alternating knots, one can also use the Jones polynomial to approximate their crossing numbers since it is known that the span (or breadth) of the Jones polynomial of a knot bounds the crossing number of the knot from below. Unfortunately, the computation of the Jones polynomial of a knot is known to be NP-hard [7]. This prevents the computation of the Jones polynomial of knots with large crossing numbers in general. For example, Knotscape, a commonly used software (developed by Hoste and Thistlethwaite [5]) for computing various knot invariants, handles knot diagrams up to about 50 crossings only. For some (non-alternating) knots constructed using the tensor products of signed graphs, it is possible to compute the Tutte polynomials of their corresponding graph tensor products easily, leading to a quicker computation of their Jones polynomials, hence a better understanding of such knots. Some such examples are provided in Section 6 . 


\section{Preliminaries}

In this section, we introduce the concept of a Tutte polynomial for a signed graph, following closely the definitions in [1]. The Tutte polynomial defined in this paper is only slightly less general than the one defined in [1] and the generalized Kauffman bracket polynomial [8, 9] may still be obtained from it by factoring.

Definition 2.1. Let $G$ be a graph with edges labelled $1,2, \ldots, n$, and let $T$ be a spanning tree of $G$. An edge $e$ of $T$ is said to be internally active if for any edge $f \neq e$ in $G$ such that $(T \backslash e) \cup f$ is a spanning tree of $G$, the label of $e$ is less than the label of $f$. Otherwise $e$ is said to be internally inactive. On the other hand, an edge $f$ of $G \backslash T$ is said to be externally active if $f$ has the smallest label among the edges in the unique cycle contained in $T \cup f$. Otherwise, $f$ is said to be externally inactive.

Example 2.2. Figure 1 shows a graph with six edges and four vertices where a labelling of the edges is given. The edges of a spanning tree $T$ are highlighted in the graph. With respect to the tree $T$, edges 1, 4 and 5 are internal edges and edges 2, 3 and 6 are external edges. Edge 1 is internally active since it has the smallest label, edge 4 is internally inactive since 4 is larger than 3 in the spanning tree 315 . Edge 5 is also internally inactive since 5 is larger than 2 in the spanning tree 142. Edge 2 is externally active since 2 is the smallest in the cycle 245. Edge 3 is externally inactive since 3 is larger than 1 in the cycle 134 and edge 6 is apparently externally inactive since 6 is the largest of all the labels.

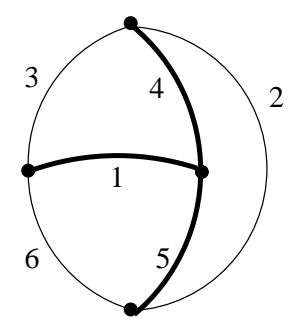

Figure 1. An example of labelled graph with a marked spanning tree.

Let $G$ be a signed (and connected) graph, that is, each edge in $G$ is assigned a + or - sign. Let $T$ be a spanning tree of $G$, for each edge $e$ in $G$ we will then assign one of the following variables to it according to the activities of $e$ (with respect to the tree $T$ ):

\begin{tabular}{|c|c|c|}
\hline sign of $e$ & activity & variable assignment \\
\hline \hline+ & internally active & $x_{+}$ \\
\hline- & internally active & $x_{-}$ \\
\hline+ & externally active & $y_{+}$ \\
\hline- & externally active & $y_{-}$ \\
\hline+ & internally inactive & $A_{+}$ \\
\hline- & internally inactive & $A_{-}$ \\
\hline+ & externally inactive & $B_{+}$ \\
\hline- & externally inactive & $B_{-}$ \\
\hline
\end{tabular}

TABLE 1. The variable assignment of an edge with respect to a spanning tree $T$. 
Definition 2.3. Let $G$ be a connected signed graph. For a spanning tree $T$ of $G$, let $C(T)$ be the product of the variable contributions from each edge of $G$ according to the variable assignment above, then the Tutte polynomial $T(G)$ is defined as the sum of all the $C(T)$ 's over all possible spanning trees of $G$.

Example 2.4. The graph given in Example 2.2 is assigned the signs as shown in Figure 2 below.

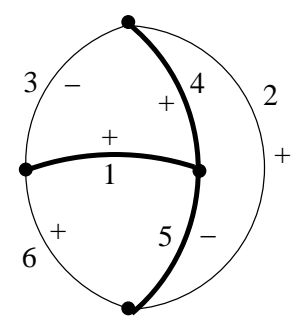

Figure 2. An example of signed and labelled graph with a marked spanning tree.

For the spanning tree shown in the figure, the total contributions of all the edges is easily calculated to be $x_{+} y_{+} A_{+} A_{-} B_{+} B_{-}$. The complete list of the spanning trees of $G$ is given in Figure 3 . We leave it for our reader to verify that the Tutte polynomial of $G$ is

$$
\begin{aligned}
& 4 x_{+} y_{+} A_{+} A_{-} B_{+} B_{-}+2 y_{+}^{2} A_{+} A_{-}^{2} B_{+}+2 x_{+}^{2} A_{+} B_{+} B_{-}^{2} \\
+ & y_{+}^{2} A_{+}^{2} A_{-} B_{-}+x_{+}^{2} A_{-} B_{+}^{2} B_{-}+y_{+}^{2} y_{-} A_{+}^{2} A_{-}+x_{+}^{2} x_{-} B_{+}^{2} B_{-} \\
+ & y_{+} A_{+} A_{-}^{2} B_{+}^{2}+y_{+} A_{+}^{3} B_{-}^{2}+x_{+} A_{-}^{2} B_{+}^{3}+x_{+} A_{+}^{2} B_{+} B_{-}^{2} .
\end{aligned}
$$

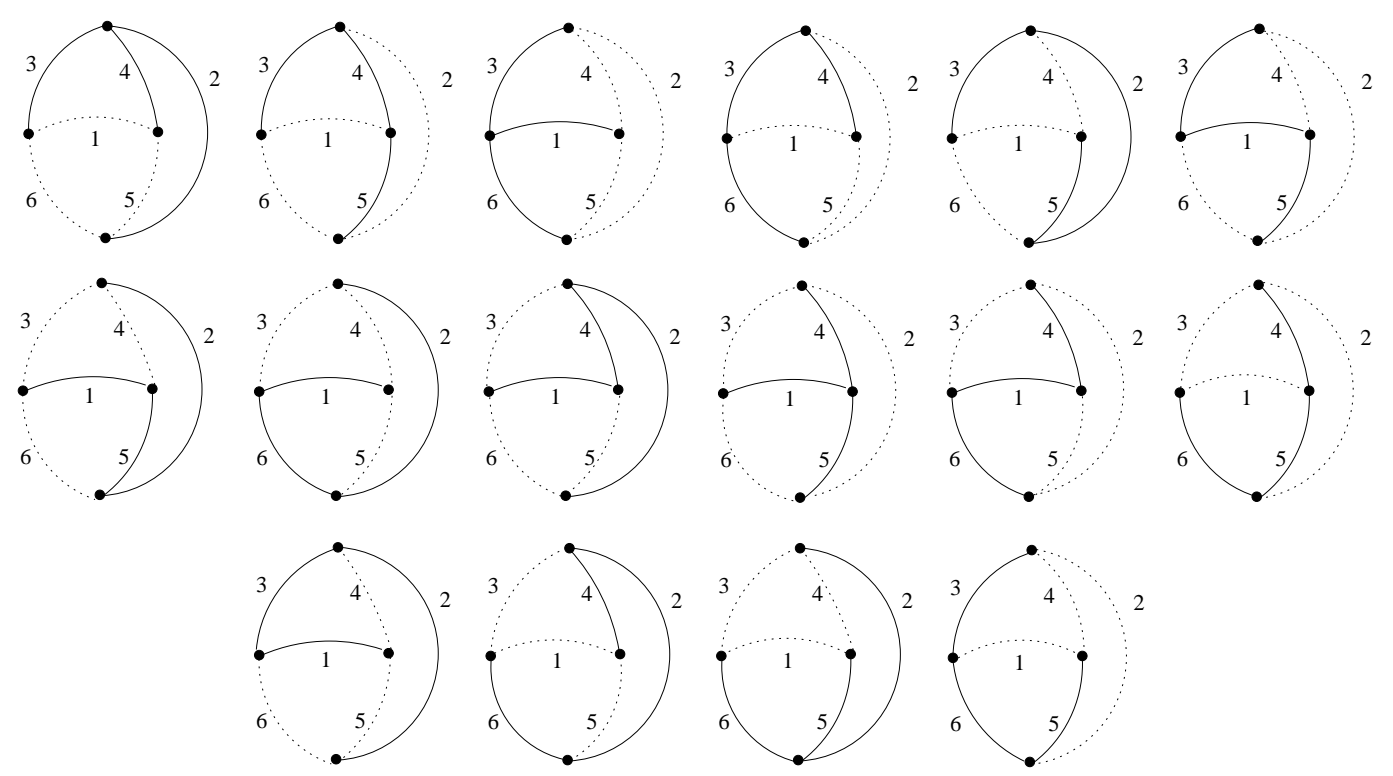

FiguRE 3. The complete set of spanning trees of the graph given in Figure 1.

Notice that the Tutte polynomial defined this way is labelling dependent. To remedy the situation, we want to factor the polynomial ring $\mathbb{Z}[\Lambda]:=\mathbb{Z}\left[A_{+}, A_{-}, B_{+}, B_{-}, x_{+}, x_{-}, y_{+}, y_{-}\right]$with an appropriate ideal $I$, such that the formula for $T(G)$ in $\mathbb{Z}[\Lambda] / I$ becomes labelling independent. An exact description 
of all such ideals (for a larger class of signed graphs) was given by Bollobás and Riordan [1, Theorem 2]. (Here we state the two-colored version.)

Theorem 2.5 (Bollobás-Riordan). Assume $I$ is an ideal of $\mathbb{Z}[\Lambda]$. Then the homomorphic image of $T(G)$ in $\mathbb{Z}[\Lambda] / I$ is independent of the labelling of the edges of $G$ if and only if

$$
y_{\nu} \operatorname{det}\left(\begin{array}{cc}
A_{+} & y_{+} \\
A_{-} & y_{-}
\end{array}\right)-y_{\nu} \operatorname{det}\left(\begin{array}{cc}
A_{+} & B_{+} \\
A_{-} & B_{-}
\end{array}\right) \in I \quad \text { for } \nu \in\{+,-\} \text {, }
$$

and

$$
x_{\nu} \operatorname{det}\left(\begin{array}{cc}
A_{+} & y_{+} \\
A_{-} & y_{-}
\end{array}\right)-x_{\nu} \operatorname{det}\left(\begin{array}{cc}
A_{+} & B_{+} \\
A_{-} & B_{-}
\end{array}\right) \in I \quad \text { for } \nu \in\{+,-\} \text {. }
$$

Bollobás and Riordan denote the ideal generated by the differences listed in Theorem 2.5 by $I_{0}$. The homomorphic image of $T(G)$ in $\mathbb{Z}[\Lambda] / I_{0}$ is the most general signed Tutte polynomial whose definition is independent of the labelling. We will describe how to factor $\mathbb{Z}[\Lambda]$ by an ideal properly containing $I_{0}$ to get the Jones polynomial. To simplify our calculations, we want to replace $I_{0}$ with a larger ideal $I_{1}$ in such a way that the effect of certain operations of signed graphs is still describable in terms of Tutte polynomials (as elements of $\mathbb{Z}[\Lambda] / I_{1}$ ). We will keep our calculations as simple as possible since we want to obtain a homomorphic image in an integral domain, and send $A_{+}, A_{-}, B_{+}, B_{-}, x_{+}, x_{-}, y_{+}$, and $y_{-}$into nonzero entries at the end. Inspired by [1, Corollary 3] we make the following definition.

Definition 2.6. We consider the signed Tutte polynomial to be an element of $\mathbb{Z}[\Lambda] / I_{1}$ where $I_{1}$ is the ideal generated by

$$
\operatorname{det}\left(\begin{array}{cc}
x_{+} & B_{+} \\
x_{-} & B_{-}
\end{array}\right)-\operatorname{det}\left(\begin{array}{cc}
A_{+} & B_{+} \\
A_{-} & B_{-}
\end{array}\right)
$$

and

$$
\operatorname{det}\left(\begin{array}{cc}
A_{+} & B_{+} \\
A_{-} & B_{-}
\end{array}\right)-\operatorname{det}\left(\begin{array}{cc}
A_{+} & y_{+} \\
A_{-} & y_{-}
\end{array}\right)
$$

Clearly $I_{1}$ properly contains $I_{0}$ so our Tutte polynomial is labelling independent. Moreover, we highlight the following observation, making [1, Corollary 3] truly useful.

Lemma 2.7. The ideal $I_{1}$ is a prime ideal. More generally, given any integral domain $R$, the ideal $I_{1}$ generated by the elements (4) and (5) in $R[\Lambda]$ is prime.

Proof. The linear map induced by $x_{+} \mapsto \widetilde{x}_{+}:=x_{+}-A_{+}, x_{-} \mapsto \widetilde{x}_{-}:=x_{-}-A_{-}, y_{+} \mapsto \widetilde{y}_{+}:=y_{+}-B_{+}$, $y_{-} \mapsto \widetilde{y}_{-}:=y_{+}-A_{+}, A_{+} \mapsto A_{+}, A_{-} \mapsto A_{-}, B_{+} \mapsto B_{+}$and $B_{-} \mapsto B_{-}$is an isomorphism between the polynomial rings $R[\Lambda]$ and $R[\widetilde{\Lambda}]:=R\left[A_{+}, A_{-}, B_{+}, B_{-}, \widetilde{x}_{+}, \widetilde{x}_{-}, \widetilde{y}_{+}, \widetilde{y}_{-}\right]$. Under this isomorphism, $I_{1}$ goes into the ideal $\widetilde{I}_{1}$ generated by

$$
\Delta_{1}:=\operatorname{det}\left(\begin{array}{cc}
\widetilde{x}_{+} & B_{+} \\
\widetilde{x}_{-} & B_{-}
\end{array}\right) \quad \text { and } \quad \Delta_{2}:=\operatorname{det}\left(\begin{array}{cc}
A_{+} & \widetilde{y}_{+} \\
A_{-} & \widetilde{y}_{-}
\end{array}\right) .
$$

We only need to show that $\widetilde{I_{1}}$ is a prime ideal in $R[\widetilde{\Lambda}]$ or, equivalently, that the factor $\operatorname{ring} R[\widetilde{\Lambda}] / \widetilde{I}_{1}$ is an integral domain. We prove the second equivalent statement in two steps. First we consider the polynomial ring $R\left[\widetilde{x}_{+}, \widetilde{x}_{-}, B_{+}, B_{-}\right]$and the ideal $\Delta_{1} \cdot R\left[\widetilde{x}_{+}, \widetilde{x}_{-}, B_{+}, B_{-}\right]$in it. This ideal is a 
determinantal ideal, and the factor $\operatorname{ring} R^{\prime}:=R\left[\widetilde{x}_{+}, \widetilde{x}_{-}, B_{+}, B_{-}\right] /\left(\Delta_{1} \cdot R\left[\widetilde{x}_{+}, \widetilde{x}_{-}, B_{+}, B_{-}\right]\right)$is a determinantal ring as defined in the book of Bruns and Vetter [2]. As a consequence of Theorem (2.10) in [2, given any integral domain $R$ and a square matrix $X$ of variables, the ideal generated by the determinant of $X$ in the polynomial ring $R[X]$ is prime. Thus $\Delta_{1} \cdot R\left[\widetilde{x}_{+}, \widetilde{x}_{-}, B_{+}, B_{-}\right]$is a prime ideal and $R^{\prime}$ is an integral domain. Observe now that $R[\widetilde{\Lambda}] / \widetilde{I_{1}}$ is isomorphic to the factor of $R^{\prime}\left[\widetilde{y}_{+}, \widetilde{y}_{-}, A_{+}, A_{-}\right]$modulo the ideal $\Delta_{2} \cdot R^{\prime}\left[\widetilde{y}_{+}, \widetilde{y}_{-}, A_{+}, A_{-}\right]$. Applying [2, Theorem (2.10)] again, this time to $R^{\prime}\left[\widetilde{y}_{+}, \widetilde{y}_{-}, A_{+}, A_{-}\right]$and $\Delta_{2} \cdot R^{\prime}\left[\widetilde{y}_{+}, \widetilde{y}_{-}, A_{+}, A_{-}\right]$, we obtain that $\Delta_{2} \cdot \widetilde{B}\left[\widetilde{y}_{+}, \widetilde{y}_{-}, A_{+}, A_{-}\right]$is a prime ideal and $R^{\prime}\left[\widetilde{y}_{+}, \widetilde{y}_{-}, A_{+}, A_{-}\right] /\left(\Delta_{2} \cdot R^{\prime}\left[\widetilde{y}_{+}, \widetilde{y}_{-}, A_{+}, A_{-}\right]\right)$is an integral domain.

Finally, since the Tutte polynomial is labelling independent under our conditions, it is easy to see that we have the following recursive formula

$$
T(G)=B_{\varepsilon} T(G \backslash e)+A_{\varepsilon} T(G / e),
$$

where $e$ is any given edge of $G$ with $\operatorname{sign} \varepsilon, G \backslash e$ is the graph obtained from $G$ by deleting $e$ and $G / e$ is the graph obtained from $G$ by contracting $e$.

Note that each spanning tree of $G$ either contains $e$ or does not contain $e$. If $e$ is in the tree, then the tree can be identified with a spanning tree of $G / e$. If $e$ is not in the tree, then the tree can be identified with a spanning tree of $G \backslash e$.

\section{The Tensor Product of Two Signed Graphs}

We will define the tensor product of two signed graphs in this section. Notice that tensor product is usually defined for matroids (see 4). Our definition of a tensor product also defines the graph only up to the underlying matroid structure. This should not represent a problem since the definition of Tutte polynomials depends only on the underlying matroid structure. All our definitions and statements may be generalized without essential adjustment to matroids just as was noted in [1, Remark 3]. Replacing each occurrence of the term "spanning tree" with "matroid basis", and understanding the words "cycle" and "dependence" in the matroid theoretic sense provides the immediate generalization. In particular, we only use the term "spanning tree" because we are interested in knot-theoretic applications where the associated graphs are connected most of the time, but our definitions and proofs work the same way for spanning forests of disconnected graphs.

Definition 3.1. Let $M$ and $N$ be two signed graphs. The positive (negative) tensor product of $M$ and $N$, denoted by $M \otimes_{+} N\left(M \otimes_{-} N\right)$, is the (signed) graph obtained by replacing each positive (negative) edge of $M$ with a copy of $N \backslash e$, where $e$ is a fixed edge of $N$ that is to be identified with the replaced edge of $M$.

Figure 4 shows an example of a positive tensor product. Notice that since there is no orientation defined on the edge $e$, the two different ways of identifying $e$ with the replaced edge in $M$ may lead to tensor products that are not isomorphic. Notice also that the sign of the edge $e$ (in the graph $N$ ) does not play any role in the tensor product since it does not appear in the tensor product. In fact, an alternative definition of the tensor product can be given by specifying two vertices of $N$ (that share the same boundary of a face of $N$ ) and replacing any positive (negative) edge of $M$ with $N$ by identifying the two vertices of the removed edge of $M$ with the two chosen vertices on $N$.

For unsigned graphs (corresponding to alternating knots), formulas for two special tensor products called the $k$-thickening and $k$-stretch may be found in [4, Lemma 6.3.24], 7, (7.2) and (7.3)], and [6, (3.8) and (3.10)]. The general formula for the general unsigned graphs can be found in [4]. Our aim is 

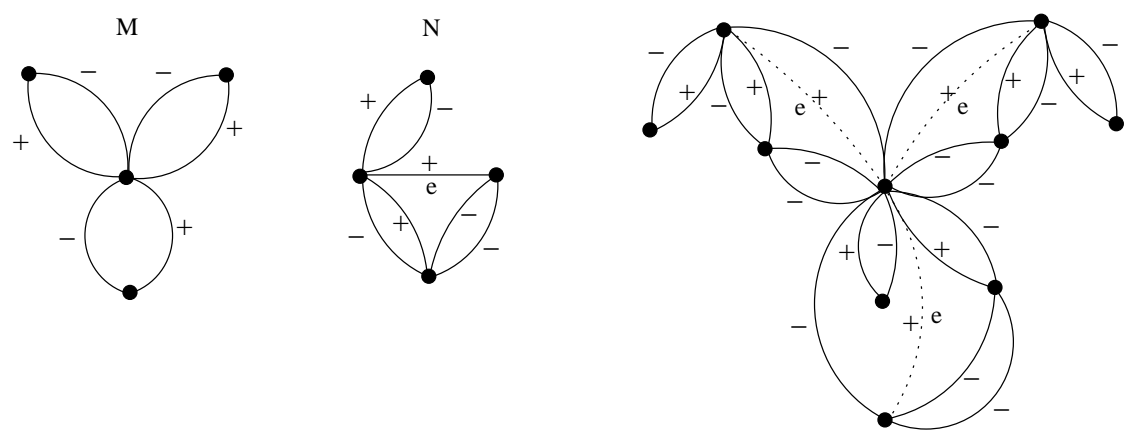

Figure 4. The positive tensor product of two signed graphs $M$ and $N$.

to extend the known results in the unsigned cases to the cases of signed graphs. Since our motivation is to apply these results in knot theory, it makes sense to look at a few examples that help one to understand what tensor products mean in knot theory. The left side of Figure 5 below shows a signed graph $M$ with four vertices, at its right side is its corresponding knot projection diagram. (The first paragraph of Section [6 discusses how to convert $M$ into its corresponding knot projection diagram.) Figure 6 shows the graph $N$ with a distinguished edge $e$, as well as its corresponding knot projection diagram. The tensor product $M \otimes_{+} N$ is the same as $M$ (so the corresponding knot projection does not change), while the tensor product $M \otimes_{-} N$ is a graph with only positive edges whose corresponding knot diagram is a reduced alternating knot projection diagram as shown in Figure 7.
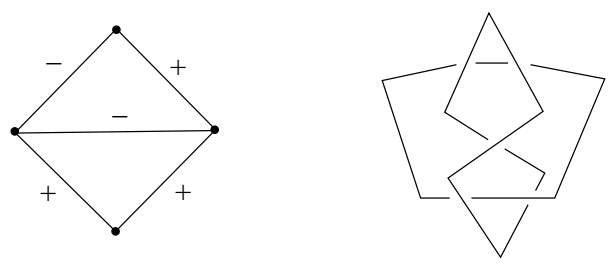

FiguRE 5. A signed graph $M$ and its corresponding non-alternating knot projection diagram.
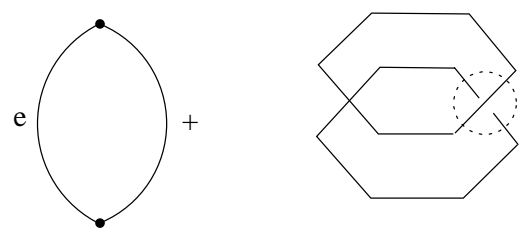

Figure 6. A signed graph $N$ with a distinguished edge and its corresponding knot projection diagram.

This shows that at the simplest level, the tensor product operation means changing the under/over strand information at the crossings to make the new knot diagram an alternating one.

\section{The polynomials $T_{C}(N, e)$ And $T_{L}(N, e)$}

In this section we introduce two polynomials defined over a signed graph $N$ with a distinguished edge $e$. These polynomials are signed generalizations of the polynomials $T_{C}$ and $T_{L}$ that occur in the paper of Jaeger, Vertigan and Welsh [7. A variant of these unsigned polynomials was first introduced 

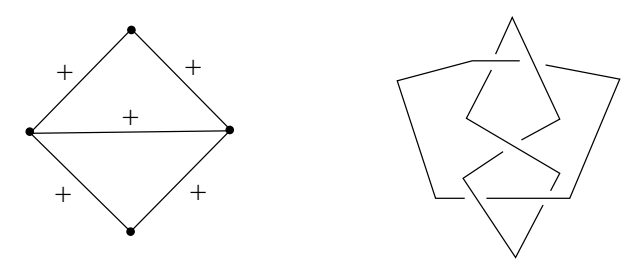

FiguRE 7. The tensor product $M \otimes_{-} N$ and its corresponding alternating knot projection diagram.

by Brylawski [3]. The definition is usually done by a system of equations. Here we make a definition that appears to be labelling dependent, in terms of activities, in the spirit of Tutte's original paper [12. We will then show that our definition is labelling independent. Our methods may be specialized to the "traditional" unsigned case, providing a new approach that appears to be closer to Tutte's original way of thinking.

For any graph $N$ with a spanning tree $P$, any edge $f$ not on $P$ will close a unique cycle in $P \cup f$. As usual in matroid theory, we will use the notation $C(P, f)$ to denote this unique cycle. As mentioned after equation (6), $P$ can be identified with a spanning tree of $N / e$ or of $N \backslash e$, depending on whether $P$ contains $e$ or not. In the following, when dealing with $N / e$ we will use the shorthand of referring to a cycle that contains the vertex contracted from $e$ as simply a cycle containing $e$.

Definition 4.1. Let $N$ be a signed graph with a distinguished edge e. Then $T_{L}(N, e)$ is the polynomial defined by the same rule that defines $T(N \backslash e)$ except that internally active edges on a cycle closed by $e$ will be considered as internally inactive instead. Similarly, $T_{C}(N, e)$ is the polynomial defined by the same rule that defines $T(N / e)$ except that externally active edges that would close a cycle containing $e$ will be considered as externally inactive instead.

Lemma 4.2. Let $N$ be a graph, $P$ be a spanning tree of $N$, e be an edge on $P, e_{i}$ and $e_{j}$ be two distinct edges not on $P$ such that $C\left(P, e_{i}\right)$ and $C\left(P, e_{j}\right)$ both contain the edge $e$. Then (1) $P_{i}=(P \backslash e) \cup e_{i}$ and $P_{j}=(P \backslash e) \cup e_{j}$ are spanning trees of $N$ as well; (2) there exists at least one cycle in $(P \backslash e) \cup e_{i} \cup e_{j}$; (3) any cycle in $(P \backslash e) \cup e_{i} \cup e_{j}$ must contain both $e_{i}$ and $e_{j}$.

Proof. (1) Since the only cycle in $P \cup e_{i}$ is $C\left(P, e_{i}\right)$ and $e$ is on this cycle, removing $e$ from $P \cup e_{i}$ results in a graph containing no cycles and having the same number of edges as $P$, so $P_{i}$ is a spanning tree of $N$. Similarly, $P_{j}$ is also a spanning tree of $N$.

(2) This is a classical graph theory result, which is equivalent to one of the circuit axioms in matroid theory.

(3) Let $C$ be a cycle contained in $(P \backslash e) \cup e_{i} \cup e_{j}$. If $C$ does not contain $e_{i}$, then it is totally contained in $P_{j}$, but that is impossible since $P_{j}$ is a tree. So $C$ must contain $e_{i}$. Similarly, $C$ must contain $e_{j}$ as well.

The following lemma is crucial in the proof of Theorem 4.4,

Lemma 4.3. The following identity holds in the ring $\mathbb{Z}[\Lambda] / I_{1}$ for all $k \geq 1$ and all $\varepsilon, \varepsilon_{1}, \ldots, \varepsilon_{k} \in$ $\{-,+\}$.

$$
A_{\varepsilon}\left(\prod_{i=1}^{k} y_{\varepsilon_{i}}-\prod_{i=1}^{k} B_{\varepsilon_{i}}\right)=\left(y_{\varepsilon}-B_{\varepsilon}\right) \sum_{i=1}^{k} A_{\varepsilon_{i}} \prod_{j=1}^{i-1} y_{\varepsilon_{j}} \prod_{t=i+1}^{k} B_{\varepsilon_{t}} .
$$


As usual, all empty products are equal to 1.

Proof. We proceed by induction on $k$. Assume first $k=1$. For $\varepsilon_{1}=\varepsilon$ the identity

$$
A_{\varepsilon}\left(y_{\varepsilon}-B_{\varepsilon}\right)=\left(y_{\varepsilon}-B_{\varepsilon}\right) A_{\varepsilon}
$$

is valid even in $\mathbb{Z}[\Lambda]$, whereas for $\varepsilon_{1} \neq \varepsilon$ the identity

$$
A_{\varepsilon}\left(y_{\varepsilon_{1}}-B_{\varepsilon_{1}}\right)=\left(y_{\varepsilon}-B_{\varepsilon}\right) A_{\varepsilon_{1}}
$$

may be rearranged as

$$
\operatorname{det}\left(\begin{array}{cc}
A_{\varepsilon_{1}} & B_{\varepsilon_{1}} \\
A_{\varepsilon} & B_{\varepsilon}
\end{array}\right)-\operatorname{det}\left(\begin{array}{cc}
A_{\varepsilon_{1}} & y_{\varepsilon_{1}} \\
A_{\varepsilon} & y_{\varepsilon}
\end{array}\right)=0
$$

and the left hand side is one of the generators of $I_{1}$. To prove the induction step, let us restate the identity in the following equivalent form.

$$
\operatorname{det}\left(\begin{array}{lll}
\sum_{i=1}^{k} A_{\varepsilon_{i}} \prod_{j=1}^{i-1} y_{\varepsilon_{j}} & \prod_{t=i+1}^{k} B_{\varepsilon_{t}} & \prod_{i=1}^{k} B_{\varepsilon_{i}} \\
A_{\varepsilon} & B_{\varepsilon}
\end{array}\right)=\operatorname{det}\left(\begin{array}{lll}
\sum_{i=1}^{k} A_{\varepsilon_{i}} \prod_{j=1}^{i-1} y_{\varepsilon_{j}} \prod_{t=i+1}^{k} B_{\varepsilon_{t}} & \prod_{i=1}^{k} y_{\varepsilon_{i}} \\
A_{\varepsilon} & y_{\varepsilon}
\end{array}\right) .
$$

Let us use $L H S_{k}$ and $R H S_{k}$ respectively as a short hand for the left hand side and right hand side of (7) respectively. Assume by induction that (7) is valid for some $k \geq 1$ and consider the identity obtained by increasing $k$ to $k+1$. The new left hand side

$$
L H S_{k+1}=\operatorname{det}\left(\begin{array}{lll}
\sum_{i=1}^{k+1} A_{\varepsilon_{i}} \prod_{j=1}^{i-1} y_{\varepsilon_{j}} & \prod_{t=i+1}^{k+1} B_{\varepsilon_{t}} & \prod_{i=1}^{k+1} B_{\varepsilon_{i}} \\
A_{\varepsilon} & B_{\varepsilon}
\end{array}\right)
$$

may be rewritten as

$$
L H S_{k+1}=\operatorname{det}\left(\begin{array}{cc}
\left(\sum_{i=1}^{k} A_{\varepsilon_{i}} \prod_{j=1}^{i-1} y_{\varepsilon_{j}} \prod_{t=i+1}^{k} B_{\varepsilon_{t}}\right) B_{\varepsilon_{k+1}}+A_{\varepsilon_{k+1}} \prod_{j=1}^{k} y_{\varepsilon_{j}} \prod_{i=1}^{k} B_{\varepsilon_{i}} \cdot B_{\varepsilon_{k+1}} \\
A_{\varepsilon} & B_{\varepsilon}
\end{array}\right),
$$

which yields the recursion

$$
L H S_{k+1}=L H S_{k} \cdot B_{\varepsilon_{k+1}}+A_{\varepsilon_{k+1}} \prod_{j=1}^{k} y_{\varepsilon_{j}} \cdot B_{\varepsilon} .
$$

Similarly, the new right hand side may be rewritten as

$$
\left.R H S_{k+1}=\operatorname{det}\left(\begin{array}{cc}
\left(\sum_{i=1}^{k} A_{\varepsilon_{i}} \prod_{j=1}^{i-1} y_{\varepsilon_{j}} \prod_{t=i+1}^{k} B_{\varepsilon_{t}}\right. \\
A_{\varepsilon}
\end{array}\right) B_{\varepsilon_{k+1}}+A_{\varepsilon_{k+1}} \prod_{j=1}^{k} y_{\varepsilon_{j}} \prod_{i=1}^{k} y_{\varepsilon_{i}} \cdot y_{\varepsilon_{k+1}}\right),
$$

yielding the recursion

$$
R H S_{k+1}=R H S_{k} \cdot B_{\varepsilon_{k+1}}+\prod_{i=1}^{k} y_{\varepsilon_{i}}\left(A_{\varepsilon_{k+1}} y_{\varepsilon}-A_{\varepsilon}\left(y_{\varepsilon_{k+1}}-B_{\varepsilon_{k+1}}\right)\right) .
$$

To show that the left hand side and the right hand side obey the same recursion formula, it is sufficient to observe that

$$
A_{\varepsilon_{k+1}} B_{\varepsilon}=A_{\varepsilon_{k+1}} y_{\varepsilon}-A_{\varepsilon}\left(y_{\varepsilon_{k+1}}-B_{\varepsilon_{k+1}}\right)
$$

holds in $\mathbb{Z}[\Lambda] / I_{1}$, which is equivalent to the already shown induction basis statement.

We will now state and prove our main result of this section. 
Theorem 4.4. Let $N$ be a signed graph with a distinguished edge $e$ and $\epsilon \in\{-1,+1\}$, then

$$
A_{\varepsilon}\left(T(N / e)-T_{C}(N, e)\right)=\left(y_{\varepsilon}-B_{\varepsilon}\right) T_{L}(N, e)
$$

and

$$
B_{\varepsilon}\left(T(N \backslash e)-T_{L}(N, e)\right)=\left(x_{\varepsilon}-A_{\varepsilon}\right) T_{C}(N, e) .
$$

Note that the equalities in the above theorem can also be expressed in terms of determinants:

$$
\operatorname{det}\left(\begin{array}{ll}
T_{L}(N, e) & T_{C}(N, e) \\
A_{\varepsilon} & B_{\varepsilon}
\end{array}\right)=\operatorname{det}\left(\begin{array}{ll}
T_{L}(N, e) & T(N / e) \\
A_{\varepsilon} & y_{\varepsilon}
\end{array}\right)
$$

and

$$
\operatorname{det}\left(\begin{array}{ll}
T_{L}(N, e) & T_{C}(N, e) \\
A_{\varepsilon} & B_{\varepsilon}
\end{array}\right)=\operatorname{det}\left(\begin{array}{ll}
T(N \backslash e) & T_{C}(N, e) \\
x_{\varepsilon} & B_{\varepsilon}
\end{array}\right)
$$

Proof. We will prove equation (8) first. Recall that $T_{L}(N, e)$ is defined by the same rule that defines $T(N \backslash e)$ except that an internally active edge with $\operatorname{sign} \varepsilon$ on a cycle closed by $e$ is considered inactive, hence contributes $A_{\varepsilon}$ instead of $x_{\varepsilon}$, and $T_{C}(N, e)$ is defined by the same rule that defines $T(N / e)$ except that an externally active edge with $\operatorname{sign} \varepsilon$ that would close a cycle containing $e$ contributes $B_{\varepsilon}$ instead of $y_{\varepsilon}$. By definition the only edges having different weight in $T(N / e)$ and $T_{C}(N, e)$ are those externally active edges on a cycle containing $e$ since the externally inactive edges are not the exceptions in the definition of $T_{C}(N, e)$ and would make the same contributions in $T(N / e)$ and $T_{C}(N, e)$. We will assign $e$ the largest index among the edges of $N$. Since $e$ does not appear in $N / e$ nor in $N \backslash e$, this will not affect our polynomials.

Let $Q$ be a spanning tree of $N$ with the property that $e \in Q$ and there exists at least one externally active edge $g$ with respect to $Q$ such that $C(Q, g)$ contains $e$. Let $S$ be the set of such $(Q, g)$ pairs. We will establish a one-to-one correspondence between $S$ and the set $\mathcal{T}_{1}$ of spanning trees of $N \backslash e$. Let $P$ be a spanning tree of $N \backslash e$. Since $e$ has the largest label, the edge $g$ with smallest label on the cycle $C(P, e)$ is different from $e$. It follows that $Q=(P \backslash g) \cup e$ is a spanning tree of $N$ and $g$ is an externally active edge with respect to $Q$. This sets up the correspondence between $P$ and the pair $(Q, g)$. We leave it to our reader to verify that this is indeed a one-to-one correspondence.

On the other hand, the spanning trees of $N / e$ can be divided into two disjoint sets: those that contain at least one externally active edge that closes a cycle containing $e$ and those that do not. Denote the first set by $\mathcal{T}_{2}$ and the second by $\mathcal{T}_{3}$. If we pair a spanning tree in $\mathcal{T}_{2}$ and an externally active edge that closes a cycle containing the vertex contracted from $e$, then apparently this will set up a one-to-one correspondence with the set $S$ as well. In other words, there is a one-to-one correspondence between $\mathcal{T}_{1}$ and the set $\mathcal{T}_{4}$ of pairs of the spanning trees of $\mathcal{T}_{2}$ and their corresponding edges described above.

Consider a spanning tree $P \in \mathcal{T}_{3}$. Since there are no externally active edges with respect to $P$, the exception rule defining $T_{C}(N, e)$ does not apply to any edge, hence the contributions of $P$ to $T(N / e)$ and $T_{C}(N, e)$ are identical. It follows that the combined contribution of $\mathcal{T}_{3}$ to the LHS is 0 and we may ignore this set entirely in the rest of our argument.

Let $P^{\prime}$ be a spanning tree of $N / e$ from the set $\mathcal{T}_{2}$ and let $e_{1}, e_{2}, \ldots, e_{k}(k \geq 1)$ be the externally active edges of $N / e$ with respect to $P^{\prime}$ such that $C\left(P^{\prime}, e_{j}\right)$ contains the vertex contracted from $e$. Let $\varepsilon_{j}$ be the sign of $e_{j}(1 \leq j \leq k)$ and let $P$ be the spanning tree of $N$ obtained from $P^{\prime}$ by recovering the edge $e$. Notice that for each $j,(P \backslash e) \cup e_{j}$ is a spanning tree of $N \backslash e$ by Lemma 4.2, Furthermore, 
$\left(P^{\prime}, e_{j}\right) \longleftrightarrow(P \backslash e) \cup e_{j}=P_{j}$ is the one-to-one correspondence between $\mathcal{T}_{1}$ and $\mathcal{T}_{4}$ discussed above. The combined contribution of $P^{\prime}$ (or of all the pairs $\left.\left(P^{\prime}, e_{j}\right), 1 \leq j \leq k\right)$ to $T(N / e)-T_{C}(N, e)$ is $\left(\prod_{j=1}^{k} y_{\varepsilon_{j}}-\prod_{j=1}^{k} B_{\varepsilon_{j}}\right)$ times the weight of the other edges of $P^{\prime}$ according to the exception rule of $T_{C}(N, e)$. Since (by Lemma 4.3)

$$
A_{\varepsilon}\left(\prod_{j=1}^{k} y_{\varepsilon_{j}}-\prod_{j=1}^{k} B_{\varepsilon_{j}}\right)=\left(y_{\varepsilon}-B_{\varepsilon}\right) \sum_{j=1}^{k} A_{\varepsilon_{j}} \prod_{i=1}^{j-1} y_{\varepsilon_{i}} \prod_{t=j+1}^{k} B_{\varepsilon_{t}}
$$

it suffices to show that, assuming that $e_{1}, e_{2}, \ldots, e_{k}$ are listed in increasing order of their labels, the contribution of $P_{j}$ to $T_{L}(N, e)$ is $A_{\varepsilon_{j}} \prod_{i=1}^{j-1} y_{\varepsilon_{i}} \prod_{t=j+1}^{k} B_{\varepsilon_{t}}$ times the weight of the other edges of $P$ (not including $e$ ) for each $j$, provided that the weight of the other edges on $P$ is the same on the LHS and RHS of the equation in the theorem.

Case 1. The edges of the cycle $C_{j}=C\left(P, e_{j}\right)=C\left(P_{j}, e\right)$ that are different from $e$ and $e_{j}$ : These edges are internal in both $P_{j}$ and $P$. On the side of $T(N / e)-T_{C}(N, e)$, these edges are internally inactive due to the fact that $e_{j}$ is externally active. On the side of $T_{L}(N, e)$, these edges are also treated as inactive edges since they are exactly the exceptions in the definition of $T_{L}(N, e)$. Thus, these edges of $P$ make the same contributions on the two sides of the equation in the theorem.

Case 2. The edges of $P$ that are not contained in the cycle $C_{j}$ : Let $f$ be such an edge. Then $f$ is either active in both $N / e$ (with respect to $P^{\prime}$ ) and $N \backslash e$ (with respect to $P_{j}$ ), or inactive in both, because it is compared to the same edges in both $N / e$ and $N \backslash e$, and none of the exceptions applies. In fact, if $f \in C\left(P^{\prime}, g\right)$ for some external edge $g$ then $g \neq e_{j}$ (otherwise $f \in C_{j}$ ), so $g$ is an external edge with respect to $P_{j}$ as well. The exception rule does not apply to $f$ on the LHS since $f$ is internal and the exception rule does not apply to $f$ on the RHS since $f \notin C_{j}$. Conversely, if $f \in C\left(P_{j}, g\right)$ for some $g$ external to $P_{j}$, then $g \neq e$ (otherwise $f \in C_{j}$ again). So $g$ is also an edge external with respect to $P_{j}$. Thus, these edges of $P$ also make the same contributions on the two sides of the equation in the theorem.

Case 3. The external edges of $N \backslash P$ with respect to $P_{j}$ that are different from the $e_{j}$ 's: Let $f$ be such an external edge. If $f$ is externally inactive in $N \backslash e$ (with respect to $P_{j}$ ), then it is also externally inactive in $N / e$ (with respect to $P^{\prime}$ ). So its weight on both sides is the same. If $f$ is externally active in $N \backslash e$ (with respect to $P_{j}$ ), then $C(P, f)$ (hence $C\left(P^{\prime}, f\right)$ ) does not contain the edge $e$ (or it would have to be one of the $e_{j}$ 's). It follows that $C\left(P^{\prime}, f\right)=C\left(P_{j}, f\right)$. So $f$ is externally active in $N / e$ with respect to $P^{\prime}$ as well. Thus $f$ makes the same contribution on both sides of the equation.

Case 4. We will now consider the weight of the edges $e_{1}, e_{2}, \ldots, e_{j+1}, \ldots, e_{k}$ in $T_{L}(N, e)$. Consider the unique cycle $C\left(P_{j}, e_{i}\right)$ for any $i \neq j$. By Lemma 4.2, $e_{i}$ and $e_{j}$ are both contained in this cycle. It follows that $e_{i}$ is externally inactive if $i>j$ by our assumption on the labels of the edges $e_{1}, e_{2}, \ldots$, $e_{k}$. On the other hand, notice that $C\left(P_{j}, e_{i}\right)$ is a subset of $C\left(P, e_{i}\right) \cup C\left(P, e_{j}\right)$. By the definition of $e_{i}$ and $e_{j}, e_{i}$ has the smallest label among the edges of $C\left(P, e_{i}\right)$ and $e_{j}$ has the smallest label among the edges of $C\left(P, e_{j}\right)$. Thus, if $i<j$, then $e_{i}$ has the smallest label among all edges of $C\left(P, e_{i}\right) \cup C\left(P, e_{j}\right)$. In particular, $e_{i}$ has the smallest label among all edges of $C\left(P_{j}, e_{i}\right)$. That is, $e_{i}$ is externally active with respect to $P_{j}$ (in $N \backslash e$ ). So the contribution of $e_{i}$ is $y_{\varepsilon_{i}}$ for any $1 \leq i<j$. It follows that the total contribution of the edges $e_{1}, e_{2}, \ldots, e_{j+1}, \ldots, e_{k}$ in $T_{L}(N, e)$ is $\prod_{i=1}^{j-1} y_{\varepsilon_{i}} \prod_{t=j+1}^{k} B_{\varepsilon_{t}}$.

Case 5. Since $e_{j}$ is an internal edge with respect to $P_{j}$ and it is on a cycle closed by $e$ (by the definition of $\left.P_{j}\right), e_{j}$ is considered to be inactive by the exception rule of $T_{L}(N, e)$. Thus the contribution of $e_{j}$ is $A_{\varepsilon_{j}}$. 
Combining the above cases finishes our proof for (8). The proof for (9) is quite similar to that of (8). However, for the sake of convenience of our reader, we still provide the details below.

$T_{L}(N, e)$ is defined by the same rule as $T(N \backslash e)$ except that internally active edges on a cycle closed by $e$ are considered as inactive instead. Thus the only edges having different weight in $T(N \backslash e)$ and $T_{L}(N, e)$ are those internally active edges on a cycle closed by $e$, since the internally inactive edges make the same contributions in $T(N \backslash e)$ and $T_{L}(N, e)$. Keep in mind that we have assigned $e$ the largest index among the edges of $N$.

Let $P$ be a spanning tree in $N \backslash e$ and let $e_{1}, e_{2}, \ldots, e_{k}(k \geq 1)$ be the internally active edges such that $P \backslash e_{j} \cup e$ is a spanning tree of $N$. Let $P_{j}^{\prime}$ be the spanning tree of $N / e$ obtained from $P$ by contracting the edge $e$ and deleting the edge $e_{j}$ from the cycle that is thus formed. Notice that $P_{j}^{\prime} \longleftrightarrow P \backslash e_{j} \cup e$ is a one-to-one correspondence. Since the edges $e_{1}, e_{2}, \ldots, e_{k}$ are on the cycle closed by $e$ in $P$, they are subject to the exception rule in the definition of $T_{L}(N, e)$, and all other edges carry the same weight in the calculations of $T_{L}(N, e)$ and $T(N \backslash e)$. Thus the contribution of $P$ to $T(N \backslash e)-T_{L}(N, e)$ is $\left(\prod_{j=1}^{k} x_{\varepsilon_{j}}-\prod_{j=1}^{k} A_{\varepsilon_{j}}\right)$ times the combined weight of the other edges. Since

$$
B_{\varepsilon}\left(\prod_{j=1}^{k} x_{\varepsilon_{j}}-\prod_{j=1}^{k} A_{\varepsilon_{j}}\right)=\left(x_{\varepsilon}-A_{\varepsilon}\right) \sum_{j=1}^{k} B_{\varepsilon_{j}} \prod_{i=1}^{j-1} x_{\varepsilon_{i}} \prod_{t=j+1}^{k} A_{\varepsilon_{t}}
$$

by Lemma 4.3, it suffices to show that, assuming that $e_{1}, e_{2}, \ldots, e_{k}$ are listed in increasing order of their labels, the contribution of $P_{j}^{\prime}$ to $T_{C}(N, e)$ is $B_{\varepsilon_{j}} \prod_{i=1}^{j-1} x_{\varepsilon_{i}} \prod_{t=j+1}^{k} A_{\varepsilon_{t}}$ times the weight of the other edges of $P_{j}^{\prime}$ for each $j$, provided that the weight of the other edges on $P$ is the same on the LHS and the RHS of equation (9) in the theorem.

If $g$ is an edge of $P$, let us denote by $K(P, g)$ the set of external edges $f$ such that $(P \backslash g) \cup f$ is a spanning tree. We have the following cases to consider.

Case 1. The edges of $K\left(P, e_{j}\right)$ that are different from $e$ and $e_{j}$. These edges are external in both $P$ and $P \backslash e_{j} \cup e$. On the side of $T(N \backslash e)-T_{L}(N, e)$, these edges are externally inactive as $e_{j}$ is internally active. On the side of $T_{C}(N, e)$, these edges are the exceptions in the definition of $T_{C}(N, e)$ hence are also considered as inactive. Hence these edges make the same contributions on both sides of the equation.

Case 2. The edges of $N \backslash P$ that are not in $K\left(P, e_{j}\right)$. Let $f$ be such an edge. Then $f$ is either inactive in both $N \backslash e$ (with respect to $P$ ) and $N / e$ (with respect to $P_{j}^{\prime}$ ) or active in both $N \backslash e$ and $N / e$, because it is compared to the same edges in both $N \backslash e$ and $N / e$. The exception rule does not apply since $f$ is external on the LHS and $e \notin C\left(P_{j}^{\prime}, f\right)$ on the RHS. Hence $f$ makes the same contribution on both sides of the equation.

Case 3. The internal edges of $P$ that are different from the $e_{i}$ 's. Let $f$ be such an edge. If $f$ is internally inactive in $N / e$ (with respect to $P_{j}^{\prime}$ ) then it is also internally inactive in $N \backslash e$ (with respect to $P$ ). If $f$ is internally active in $N / e$ then $K\left(P_{j}^{\prime}, f\right)$ does not contain the vertex contracted from $e$ (otherwise it would be one of the $e_{i}$ 's). It follows that $K(P, f)=K\left(P \backslash e_{j} \cup e, f\right)$, so $f$ is internally active with respect to $P$ as well. Thus $f$ makes the same contribution on both sides of the equation.

Case 4. We now consider the edges $e_{1}, e_{2}, \ldots, e_{k}$ in $T_{C}(N, e)$. Recall that $P_{j}^{\prime}$ is the spanning tree of $N / e$ obtained from $P$ by contracting the edge $e$ and deleting the edge $e_{j}$. Consider the cut $K\left(P_{j}^{\prime}, e_{i}\right)$ for any $i \neq j . e_{i}$ and $e_{j}$ are both contained in this cut. It follows that $e_{i}$ is internally inactive if $i>j$, by our assumption on the labels of the edges $e_{1}, e_{2}, \ldots, e_{k}$, and that $e_{i}$ is internally active if $i<j$. So 
the contribution of $e_{i}$ is $x_{\varepsilon_{i}}$ for any $1 \leq i<j$ and $A_{\varepsilon_{i}}$ for $j<i \leq k$. Finally, since $e_{j}$ is external to $P_{j}^{\prime}$ and it closes a cycle in $P_{j}^{\prime}$ containing $e$, the exception rule for $T_{C}(N, e)$ applies to it. So it is always considered as inactive hence its contribution in $T_{C}(N, e)$ is $B_{\varepsilon_{j}}$. It follows that the total contribution of $e_{1}, e_{2}, \ldots, e_{k}$ in $T_{C}(N, e)$ is $B_{\varepsilon_{j}} \prod_{i=1}^{j-1} x_{\varepsilon_{i}} \prod_{t=j+1}^{k} A_{\varepsilon_{t}}$, as desired.

One of the most important consequences of Theorem 4.4 is the following.

Corollary 4.5. The polynomials $T_{C}(N, e)$ and $T_{L}(N, e)$ are independent of the labelling. They may be equivalently defined by the system of equations (8) and (9).

In fact, by Theorem 4.4, for any $\varepsilon \in\{+,-\}$, setting $z_{C}=T_{C}(N, e)$ and $z_{L}=T_{L}(N, e)$ provides a solution of the linear system of equations

$$
\begin{aligned}
\left(y_{\varepsilon}-B_{\varepsilon}\right) z_{L}+A_{\varepsilon} z_{C} & =A_{\varepsilon} T(N / e) \\
B_{\varepsilon} z_{L}+\left(x_{\varepsilon}-A_{\varepsilon}\right) z_{C} & =B_{\varepsilon} T(N \backslash e) .
\end{aligned}
$$

Here $z_{L}$ are $z_{C}$ are the unknowns, and the givens belong to $\mathbb{Z}[\Lambda] / I_{1}$, an integral domain by Lemma 2.7 Cramer's rule is applicable in the quotient field of $\mathbb{Z}[\Lambda] / I_{1}$, and we have

$$
\operatorname{det}\left(\begin{array}{ll}
y_{\varepsilon}-B_{\varepsilon} & A_{\varepsilon} \\
B_{\varepsilon} & x_{\varepsilon}-A_{\varepsilon}
\end{array}\right)=x_{\varepsilon} y_{\varepsilon}-A_{\varepsilon} y_{\varepsilon}-B_{\varepsilon} x_{\varepsilon}
$$

which is a nonzero element of $\mathbb{Z}[\Lambda] / I_{1}$, independently of the choice of $\varepsilon$, since each element of $I_{1}$ is a $\mathbb{Z}$-linear combination of monomials involving both negative and positive variables.

We may recover the classical unsigned case that is widely studied in the literature in two steps as follows. Consider unsigned graphs as special signed graphs whose edges are all positive. The signed Tutte polynomial of such a graph may be considered as a polynomial in the positive variables only. The subring of $\mathbb{Z}[\Lambda] / I_{1}$, generated by the integers and the positive variables is easily seen to be isomorphic to the polynomial ring $\mathbb{Z}\left[x_{+}, y_{+}, A_{+}, B_{+}\right]$, because $I_{1}$ involves only polynomials with terms of mixed signature, as mentioned above. We thus consider $T_{C}(N, e)$ and $T_{L}(N, e)$ as elements of $\mathbb{Z}\left[x_{+}, y_{+}, A_{+}, B_{+}\right]$, defined by the exception rules given in Definition 4.1. Under these circumstances Lemma 4.3 may be replaced with the trivial identity

$$
A_{+}\left(y_{+}^{k}-B_{+}^{k}\right)=A_{+}\left(y_{+}-B_{+}\right) \cdot \sum_{i=1}^{k} y_{+}^{i-1} B_{+}^{k-i},
$$

and Theorem 4.4 specializes to the following statement.

Theorem 4.6. Given an unsigned graph $N$ with a distinguished edge e, the signed polynomials $T_{C}(N, e), T_{L}(N, e) \in \mathbb{Z}\left[x_{+}, y_{+}, A_{+}, B_{+}\right]$satisfy

$$
A_{+}\left(T(N / e)-T_{C}(N, e)\right)=\left(y_{+}-B_{+}\right) T_{L}(N, e)
$$

and

$$
B_{+}\left(T(N \backslash e)-T_{L}(N, e)\right)=\left(x_{+}-A_{+}\right) T_{C}(N, e) .
$$

The system of equations in Theorem 4.6 uniquely determines the polynomials $T_{C}(N, e), T_{L}(N, e) \in$ $\mathbb{Z}\left[x_{+}, y_{+}, A_{+}, B_{+}\right]$, and may be used as their alternative definition. Consider finally the ring homomorphism $\phi: \mathbb{Z}\left[x_{+}, y_{+}, A_{+}, B_{+}\right] \rightarrow \mathbb{Z}[x, y]$, given by $x_{+} \mapsto x, y_{+} \mapsto y, A_{+} \mapsto 1$, and $B_{+} \mapsto 1$. On the one hand $T_{C}(N, e), T_{L}(N, e)$ go into polynomials that may be defined by modifying the definition of the ordinary Tutte polynomials $T(N \backslash e ; x, y)$ and $T(N / e ; x, y)$ according to the exception rules given in Definition 4.1. On the other hand these homomorphic images satisfy the homomorphic images of the equations given in Theorem 4.6 which still has a unique solution in the polynomial ring $\mathbb{Z}[x, y]$. 
Corollary 4.7. Given an unsigned graph $N$ with a distinguished edge e, let us define the polynomials $T_{C}(N, e)$ and $T_{L}(N, e)$ by modifying the definition of the ordinary Tutte polynomials $T(N \backslash e ; x, y)$ and $T(N / e ; x, y)$ according to the exception rules given in Definition 4.1. Then these polynomials may be equivalently defined by the system of equations

$$
\begin{aligned}
& T(N / e)-T_{C}(N, e)=(y-1) T_{L}(N, e) \\
& T(N \backslash e)-T_{L}(N, e)=(x-1) T_{C}(N, e) .
\end{aligned}
$$

In particular, the definition is labelling independent.

The system of equations (12) appears as the definition of the unsigned polynomials $T_{C}$ and $T_{L}$ in [7, Equation (4.2)].

\section{The Tutte polynomial of a signed tensor Product}

The main result of this section is that the Tutte polynomial of the tensor product $M \otimes_{+} N$ or $M \otimes_{-} N$ can be computed using the Tutte polynomial $T(M)$ of $M$ and the special Tutte polynomials $T(N \backslash e)$, $T(N / e), T_{C}(N, e)$ and $T_{L}(N, e)$ of $N$ discussed in the last section by simple variable substitutions. First we need the following lemma.

Lemma 5.1. Let $N$ be a signed graph with a distinguished edge e. Then the endomorphism of $\mathbb{Z}[\Lambda]$ given by

$$
x_{+} \mapsto T(N \backslash e) \quad A_{+} \mapsto T_{L}(N, e) \quad y_{+} \mapsto T(N / e) \quad B_{+} \mapsto T_{C}(N, e)
$$

(with all negative variables unchanged) sends $I_{1}$ into itself. Similarly, the endomorphism of $\mathbb{Z}[\Lambda]$ given by

$$
x_{-} \mapsto T(N \backslash e) \quad A_{-} \mapsto T_{L}(N, e) \quad y_{-} \mapsto T(N / e) \quad B_{-} \mapsto T_{C}(N, e)
$$

(with all positive variables unchanged) sends $I_{1}$ into itself as well. Consequently, these endomorphisms induce endomorphisms of the factor ring $\mathbb{Z}[\Lambda] / I_{1}$.

Proof. This is immediate from Equations (10) and (11), showing that the given endomorphisms send the two generators of $I_{1}$ into elements of $I_{1}$.

Theorem 5.2. Let $M$ be a signed graph and $N$ a signed graph with a distinguished edge e. Then $T\left(M \otimes_{+} N\right)$ can be computed from $T(M)$ by keeping the negative variables unchanged and using the substitutions

$$
x_{+} \mapsto T(N \backslash e) \quad A_{+} \mapsto T_{L}(N, e) \quad y_{+} \mapsto T(N / e) \quad B_{+} \mapsto T_{C}(N, e) .
$$

Similarly, $T\left(M \otimes_{-} N\right)$ can be computed from $T(M)$ by keeping the positive variables unchanged and using the substitutions

$$
x_{-} \mapsto T(N \backslash e) \quad A_{-} \mapsto T_{L}(N, e) \quad y_{-} \mapsto T(N / e) \quad B_{-} \mapsto T_{C}(N, e) .
$$

Proof. By Lemma 5.1, the given substitutions induce an endomorphism of $\mathbb{Z}[\Lambda] / I_{1}$. This means that the operation is well-defined in the sense that no matter which representative of $T(M), T(N \backslash e)$, $T(N / e), T_{C}(N, e)$ and $T_{L}(N, e)$ we use, the resulting polynomial will belong to the same equivalence class of $\mathbb{Z}[\Lambda]$ modulo $I_{1}$. Thus we need only to show that one specific representative of $T\left(M \otimes_{+} N\right)$ and $T\left(M \otimes_{-} N\right)$ can be computed from one specific representative of $T(M), T(N \backslash e), T(N / e), T_{C}(N, e)$ and $T_{L}(N, e)$ by the above substitutions under one special labelling rule. We will show this for $T\left(M \otimes_{+} N\right)$. The proof for $T\left(M \otimes_{-} N\right)$ is exactly the same. 
Assume that $M$ has $m$ edges and $N \backslash e$ has $n$ edges and both have been assigned a labelling using positive integers from 1 to $m$ and 1 to $n$ respectively. For the sake of convenience, an edge of $M$ with label $i$ will be called $e_{i}$ in this proof. We will now label $M \otimes_{+} N$ in the following way. First, any negative edge in $M$ (which is not affected by the tensor operation in $M \otimes_{+} N$ ) will retain its original label index. On the other hand, after a positive edge with index $i$ is replaced by $N \backslash e$, the edge in this copy of $N \backslash e$ with original label index $j$ will now be assigned label index $i+\frac{j}{n+1}$. For the sake of convenience, we may denote by $N_{i}$ the copy of $N \backslash e$ that replaces the edge in $M$ with label $i$ (as a part of the graph $\left.M \otimes_{+} N\right)$. Under this labelling, if $e_{j}$ is a negative edge in $M$ (so it will not be replaced in $T\left(M \otimes_{+} N\right)$ by $\left.N \backslash e\right)$, then the label of any edge in $N_{i}$ is less than $j$ if $i<j$, and is larger than $j$ if $i>j$. This same rule applies to edges from $N_{i}$ and $N_{j}$ as well.

Let us now consider a spanning tree $P^{\prime}$ of $M \otimes_{+} N$. Notice that $P^{\prime}$ induces a spanning tree $P$ of $M$ in a natural way: if $P^{\prime}$ contains an edge of $M$, then that edge is retained. If $P^{\prime}$ contains a path in $N_{i}$ that connects the two vertices of the edge $e_{i}$ in $M$ replaced by $N_{i}$, then that path is replaced by $e_{i}$, otherwise the edges of $N_{i}$ will simply be removed. On the other hand, a spanning tree $P$ of $M$ can be extended to a spanning tree of $M \otimes_{+} N$ by replacing a positive edge $e_{i}$ in $P$ by a spanning tree of $N$ (notice that this spanning tree may contain the edge $e$ and in this case the resulting spanning tree $P^{\prime}$ of $M \otimes_{+} N$ would not contain a path connecting the two vertices of the edge $e_{i}$ ). Any spanning tree $P^{\prime}$ of $M \otimes_{+} N$ obtained from the spanning tree $P$ of $M$ is called an offspring of $P$ and the tree $P$ is called a parent tree of $P^{\prime}$. We will now consider the total contribution of all the offsprings of a parent tree $P$ in $M$.

Case 1. The negative edges of $M \otimes_{+} N$ that come directly from $M$. Let $f$ be such an edge and let $P^{\prime}$ be an offspring of $P$. Apparently, $f$ is internal (external) to $P$ if and only if it is internal (external) to $P^{\prime}$. Furthermore, the activity of $f$ in $P^{\prime}$ is the same as its activity in $P$ by the choice of our labelling for $M \otimes_{+} N$. Thus $f$ makes the same contribution in $T(M)$ and $T\left(M \otimes_{+} N\right)$. In other words, we do not replace the negatively indexed variables in $T(M)$ when computing $T\left(M \otimes_{+} N\right)$.

Case 2. A positive edge $e_{i}$ in $M$ that is internally active with respect to $P$. This edge is replaced by $N_{i}$. Notice that any offspring $P^{\prime}$ of $P$ induces a spanning tree $P_{i}$ of $N$ that does not contain $e$, that is, a spanning tree $P_{i}$ of $N \backslash e$. In other words, $P^{\prime}$ contains a path (in $N_{i}$ ) connecting the two vertices of the edge $e_{i}$. Any internally active edge $f$ of $N_{i}$ with respect to $P_{i}$ is internal to $P^{\prime}$ and is also active since any edge $g$ such that $\left(P^{\prime} \backslash f\right) \cup g$ is a spanning tree of $M \otimes_{+} N$ will have a larger label by the labelling of $M \otimes_{+} N$. Of course, an internally inactive edge $f$ of $N_{i}$ with respect to $P_{i}$ is an internally inactive edge in $M \otimes_{+} N$ with respect to $P^{\prime}$. On the other hand, an externally active edge $f$ of $N_{i}$ with respect to $P_{i}$ is external to $P^{\prime}$ since $C\left(P^{\prime}, f\right)=C\left(P_{i}, f\right)$ and the labelling of $N_{i}$ inherits the relative ordering of the labelling of $N \backslash e$ by our labelling choice. It follows that the activity of $f$ in $N_{i}$ is the same as its activity in $M \otimes_{+} N$. Therefore, the total contribution of $N_{i}$ to $T\left(M \otimes_{+} N\right)$ with respect to any given parent spanning tree $P$ is $T(N \backslash e)$. That is, we may replace $x_{+}$in $T(M)$ by $T(N \backslash e)$ when computing $T\left(M \otimes_{+} N\right)$.

Case 3. A positive edge $e_{i}$ in $M$ that is internally inactive with respect to $P$. Again such an edge is replaced by $N_{i}$ and $P^{\prime}$ contains a path (in $N_{i}$ ) connecting the two vertices of the edge $e_{i}$. If $f$ is an edge on this path, then $f$ is inactive since there exists an edge $g$ of $M \otimes_{+} N$ with label smaller than that of $f$ such that $\left(P^{\prime} \backslash f\right) \cup g$ is a spanning tree of $M \otimes_{+} N$, see Figure 8 , The top of Figure 8 shows the graph of $P$, with $e_{i} \in P, e_{j} \notin P$, and $j<i$. Thus $e_{i}$ is internally inactive. The bottom left of Figure 8 shows the case when $e_{j}$ is negative and is not replaced by $N \backslash e$. In this case, $\left(P^{\prime} \backslash f\right) \cup e_{j}$ is a spanning tree of $M \otimes_{+} N$ with the label $e_{j}$ less than the label of $f$ (which is between $i$ and $i+1$ by our choice of labelling on $\left.M \otimes_{+} N\right)$. The bottom right of Figure 8 shows the case when $e_{j}$ is positive and is replaced by $N_{j}=N \backslash e$. In this case there exists some edge $f_{j}$ in $N_{j}, f_{j} \notin P^{\prime}$, such that $\left(P^{\prime} \backslash f\right) \cup f_{j}$ 
is a spanning tree of $M \otimes_{+} N$, and again the label of $f_{j}$ (between $j$ and $j+1$ ) is less than the label of $f$ (between $i$ and $i+1$ ). To summarize: an internally active edge $f$ in $N_{j}$ with respect to $P_{j}$ is internally active in $M \otimes_{+} N$ with respect to $P^{\prime}$ if and only if it is not on $C\left(P_{j}, e\right)$. Finally, the activity of any edge $f$ of $N_{j}$ external to $P^{\prime}$ is decided within $N_{j}$ since the unique cycle $C\left(P^{\prime}, f\right)=C\left(P_{j}, f\right)$ is contained in $N_{j}$. These rules are exactly the ones that define the polynomial $T_{L}(N, e)$, hence we may replace $A_{+}$in $T(M)$ by $T_{L}(N, e)$ when computing $T\left(M \otimes_{+} N\right)$.
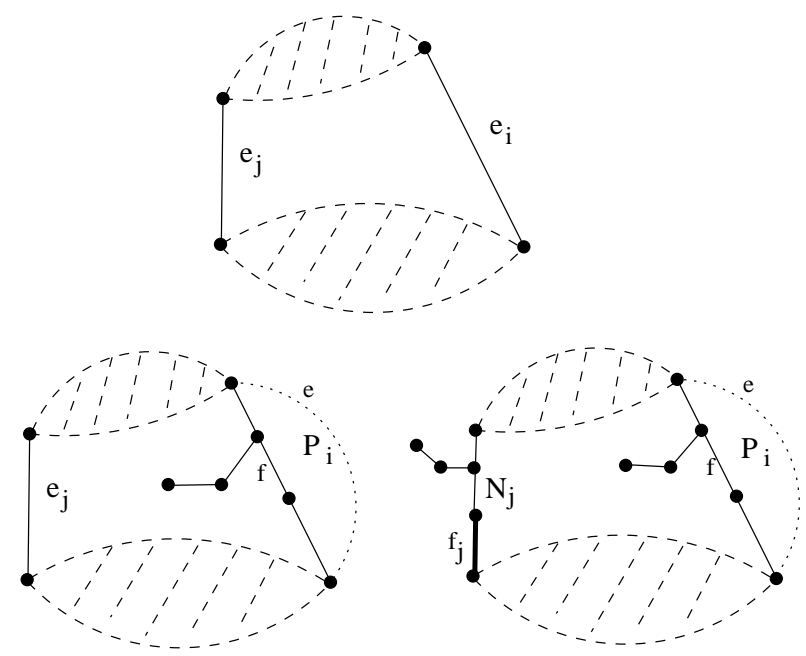

Figure 8. The activities of an internal edge in $N_{i}$ when $e_{i}$ is internally inactive in $M$.

Case 4. A positive edge $e_{i}$ in $M$ that is externally active with respect to $P$. Let $N_{i}$ be the copy of $N \backslash e$ replacing $e_{i}$ with our labelling defined earlier and let $P^{\prime}$ be an offspring of $P$. Since $e_{i}$ is not on $P, P^{\prime}$ does not contain a path (in $N_{i}$ ) that connects the two vertices of $e_{i}$. It follows that $P^{\prime}$ induces a spanning tree $P_{i}^{\prime}$ in $N / e$. Let $f$ be an internal edge on $P_{i}^{\prime}$. Then $P_{i}^{\prime} \backslash f$ can be made into a tree only by adding an edge in $N_{i}$. In other words, the activity of $f$ is decided "locally" in $N / e$ with respect to the spanning tree $P_{i}^{\prime}$. On the other hand, if $f$ is an external edge in $M \otimes_{+} N$ with respect to $P^{\prime}$, then the cycle $C\left(P^{\prime}, f\right)$ is contained in $N_{i}$ and can be considered as a cycle of $N / e$. Thus the activity of $f$ in $M \otimes_{+} N$ with respect to $P^{\prime}$ is the same as the activity of $f$ in $N / e$ with respect to $P_{i}^{\prime}$. It follows that the total contribution of the edges of $N_{i}$ (over all possible $P^{\prime}$ ) is the same as $T(N / e)$. Hence we may replace $y_{+}$in $T(M)$ by $T(N / e)$ when computing $T\left(M \otimes_{+} N\right)$.

Case 5. A positive edge $e_{i}$ in $M$ that is externally inactive with respect to $P$. Let $N_{i}$ be the copy of $N \backslash e$ replacing $e_{i}$ with our labelling defined earlier and let $P^{\prime}$ be an offspring of $P$. Again, $P^{\prime}$ does not contain a path (in $N_{i}$ ) that connects the two vertices of $e_{i}$ hence it induces a spanning tree $P_{i}^{\prime}$ in $N / e$. As in case 4 above, if $f$ is an external edge in $M \otimes_{+} N$ with respect to $P^{\prime}$, then the cycle $C\left(P^{\prime}, f\right)$ is contained in $N_{i}$ and can be considered as a cycle of $N / e$. Thus the activity of $f$ in $M \otimes_{+} N$ with respect to $P^{\prime}$ is the same as the activity of $f$ in $N / e$ with respect to $P_{i}^{\prime}$. On the other hand, if $f$ is an internal edge in $M \otimes_{+} N$ with respect to $P^{\prime}$, then $f$ is internal in $N / e$ with respect to $P_{i}^{\prime}$. If $f$ is inactive in $N / e$ locally with respect to $P_{i}^{\prime}$, then it is inactive. If the addition of $f$ to $P^{\prime}$ completes a path connecting the two vertices of $e_{i}$, then there exists an edge in $M \otimes_{+} N$ (but not in $N_{i}$ ) with a smaller label that is on the cycle $C\left(P^{\prime}, f\right)$. In any case, $f$ is still inactive in $M \otimes_{+} N$. Finally, if $f$ is internally active in $N / e$ with respect to $P_{i}^{\prime}$, then it will still be internally active in $M \otimes_{+} N$ if the cycle $C\left(P^{\prime}, f\right)$ is contained in $N_{i}$, otherwise $f$ would be inactive in $M \otimes_{+} N$. But this means exactly that any externally active edge $f$ such that $e$ is on the cycle $C\left(P_{i}^{\prime \prime}, f\right)$ in $N$ is to be considered as 
externally inactive in $M \otimes_{+} N$ (here $P_{i}^{\prime \prime}$ is the spanning tree of $N$ obtained from $P_{i}^{\prime}$ by recovering the edge $e$ ). It follows that the total contribution of the edges of $N_{i}$ (over all possible $P^{\prime}$ ) is the same as $T_{C}(N, e)$. Hence we may replace $B_{+}$in $T(M)$ by $T_{C}(N, e)$ when computing $T\left(M \otimes_{+} N\right)$. This finishes our proof.

We conclude this section by outlining how Theorem 5.2 and its proof may be specialized to the unsigned case. Just like at the end of Section 4, consider unsigned graphs as special signed graphs whose edges are all positive, and all polynomial invariants as elements of the polynomial ring $\mathbb{Z}\left[x_{+}, y_{+}, A_{+}, B_{+}\right]$. Given an unsigned graph $M$ and an unsigned graph $N$ with a distinguished edge $e$, considered as signed graphs having positive edges only, the unsigned tensor product $M \otimes N$ is identifiable with the signed tensor product $M \otimes_{+} N$. (For a definition of the unsigned tensor product see [3], 4], 6], or [7, we will use the above observation as our definition.) We may adapt the proof of Theorem 5.2 in such a way that negative variables never appear in the picture, and obtain the following analogous statement.

Theorem 5.3. Let $M$ be an unsigned graph and $N$ an unsigned graph with a distinguished edge $e$. Then $T\left(M \otimes_{+} N\right) \in \mathbb{Z}\left[x_{+}, y_{+}, A_{+}, B_{+}\right]$can be computed from $T(M) \in \mathbb{Z}\left[x_{+}, y_{+}, A_{+}, B_{+}\right]$by using the substitutions

$$
x_{+} \mapsto T(N \backslash e) \quad y_{+} \mapsto T(N / e) \quad A_{+} \mapsto T_{L}(N, e) \quad B_{+} \mapsto T_{C}(N, e)
$$

Observe now that the signed Tutte polynomial of $M$, when written as a sum of monomials in positive variables, has the property that the total degree in $x_{+}$and $A_{+}$of each monomial is the total number of internal edges of some spanning tree, a.k.a. the $\operatorname{rank} r(M)$ of the matroid $M$, whereas the total degree in $y_{+}$and $B_{+}$is the total number of external edges, that is, $|M|-r(M)$. After extending $\mathbb{Z}\left[x_{+}, y_{+}, A_{+}, B_{+}\right]$to its localization $\mathbb{Z}\left[x_{+}, y_{+}, A_{+}, B_{+}\right]_{S}$ by the semigroup $S$ generated by $\left\{T_{C}(N, e), T_{L}(N, e)\right\}$, Theorem 5.3 may be rephrased as follows.

Corollary 5.4. Let $M$ be an unsigned graph and $N$ an unsigned graph with distinguished edge e. Then $T\left(M \otimes_{+} N ; x_{+}, y_{+}, A_{+}, B_{+}\right) \in \mathbb{Z}\left[x_{+}, y_{+}, A_{+}, B_{+}\right]_{S}$ is given by

$$
T\left(M \otimes_{+} N\right)=T_{L}(N, e)^{r(M)} T_{C}(N, e)^{|M|-r(M)} \cdot T\left(M ; T(N \backslash e) / T_{L}(N, e), T(N / e) / T_{C}(N, e), 1,1\right) .
$$

Here all polynomials are considered as elements of $\mathbb{Z}\left[x_{+}, y_{+}, A_{+}, B_{+}\right]_{S}$.

It should be noted that for a nontrivial graph $N$ with a distinguished edge $e$, the polynomials $T_{C}(N, e)$ and $T_{C}(N, e)$ are not zero since they may be computed as a sum of monomials of positive coefficients. This new substitution rule has the property that the contribution of the inactive edges of $M$ is 1 , hence the formula "factors" through using the ordinary Tutte polynomial of $M$. More precisely, we may uniquely extend the homomorphism $\phi: \mathbb{Z}\left[x_{+}, y_{+}, A_{+}, B_{+}\right] \rightarrow \mathbb{Z}[x, y]$ given by $x_{+} \mapsto x, y_{+} \mapsto y$, $A_{+} \mapsto 1, B_{+} \mapsto 1$ to a homomorphism $\mathbb{Z}\left[x_{+}, y_{+}, A_{+}, B_{+}\right]_{S} \rightarrow \mathbb{Z}[x, y]_{\phi(S)}$ and apply it to both sides of the equation in Corollary 5.4. Observe that both sides belong to the subring $\mathbb{Z}[x, y]$, only some of the calculation on the right hand side needs to be performed in $\mathbb{Z}[x, y]_{\phi(S)}$. Thus we obtain a new proof of the following classical result:

Corollary 5.5. Let $M$ be an unsigned graph and $N$ an unsigned graph with a distinguished edge $e$. Then the ordinary Tutte polynomial $T(M \otimes N) \in \mathbb{Z}[x, y]$ may be obtained from the ordinary Tutte polynomial $T(M) \in \mathbb{Z}[x, y]$ by substituting $T(N \backslash e) / T_{L}(N, e)$ into $x, T(N / e) / T_{C}(N, e)$ into $y$, and multiplying the resulting rational expression with $T_{L}(N, e)^{r(M)} T_{C}(N, e)^{|M|-r(M)}$. Here $T_{C}(N, e)$ and $T_{L}(N, e)$ are elements of $\mathbb{Z}[x, y]$, defined by (12). 
This result may be found in [7, Equation (4.1)] without a proof. The first published proof of a differently phrased but equivalent statement is due to Brylawski [3], which appears to take a very different approach. It should be noted that we referred to our signed results only for brevity's sake, the shortest direct proof of Corollary 5.5 may be obtained by directly adapting the proof of Theorem 5.2 to the unsigned case. We leave the details to the reader.

\section{Applichtions to KNOT THEORY}

It is well known that the Jones polynomial of a knot $K$ can be obtained from the Tutte polynomial of the dual graph of $D$, where $D$ is a regular projection of $K$ 9. Let us first give a brief description of this process. One starts from a regular projection $D$ of the knot $K$. We then shade the regions in its projection either "white" or "dark" in a checkerboard fashion, so that no two dark regions are adjacent, and no two white regions are adjacent. We usually consider the infinite region surrounding the knot projection to be white. Note that as we move diagonally over a knot crossing, we go from a white region to a white region, or from a dark region to a dark region. Next we construct a dual graph of $D$ by converting the dark regions in $D$ into vertices in a graph $G$ and converting the crossings in $D$ between two dark regions into edges incident to the corresponding vertices in $G$. So if we can move diagonally over a knot crossing from one dark region to another, then these two dark regions and the crossing will be represented in $G$ as two vertices connected by an edge. Note that we may obtain parallel edges from some knot projections. Now we have our unsigned graph. To obtain the signed version, we look at each crossing in the knot projection. If, after the upper strand passes over the lower, the dark region is to the left of the upper strand, then we denote this as a positive crossing. If the dark region is to the right of the upper strand, we denote it as a negative crossing. Then our signed graph is obtained by marking each edge of $G$ with the same sign as the crossing of $K$ to which it corresponds. See Figure 9 .
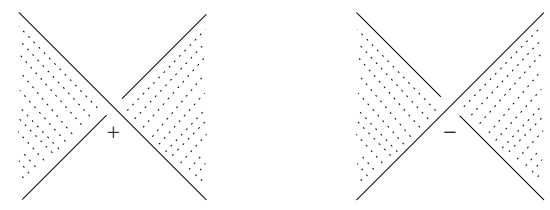

Figure 9. The assignment of signs at a crossing (vertex) for the graph $G$.

The following theorem is due to Kauffman [8, 9].

Theorem 6.1. Let $G$ be the (signed) dual graph of a regular knot projection $D$ of $K$ as described above, then $T(G)$ equals the Kauffman bracket polynomial $\langle K\rangle$ under the following variable substitutions:

$$
\begin{aligned}
& x_{+} \mapsto-A^{-3}, x_{-} \mapsto-A^{3}, y_{+} \mapsto-A^{3}, y_{-} \mapsto-A^{-3} \\
& A_{+} \mapsto A, A_{-} \mapsto A^{-1}, B_{+} \mapsto A^{-1}, B_{-} \mapsto A .
\end{aligned}
$$

Furthermore, the Jones polynomial $V_{K}(t)$ of $K$ can be obtained from

$$
V_{K}(t)=\left(-A^{-3}\right)^{w(K)}\langle K\rangle
$$

by setting $A=t^{-\frac{1}{4}}$, where $w(K)$ is the writhe of the projection $D$.

It is thus possible for us to use Theorem 5.2 to compute the Jones polynomials for some large non-alternating knots. We will demonstrate this by a few examples. We will first do this for a small knot so we can compare our result with the direct computation result using an existing software. We will then do this for a much larger knot beyond the capacity of the existing programs. 
Example 6.2. In this example, let us use the signed graphs obtained from the standard minimal knot diagrams of the non-alternating knot $9_{49}$ and the alternating knot $4_{1}$ as $M$ and $N$ respectively, see Figure 10. Notice that the identifying edge $e$ is marked in the figure. Since the tensor product is
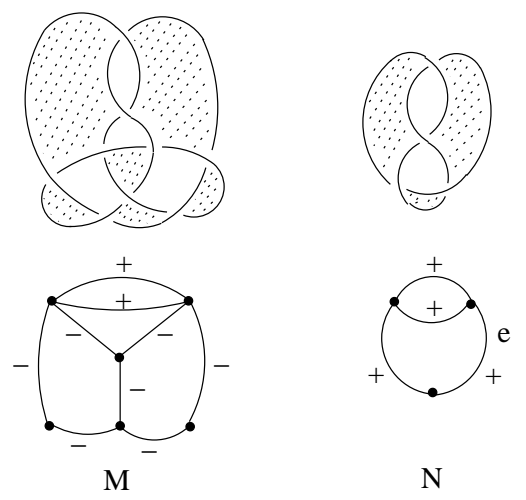

Figure 10. The knots $9_{49}, 4_{1}$ and their corresponding signed graphs.

not unique, it is possible for us to get several different knots from $M \otimes_{+} N$. One of such is shown in Figure 11, The Tutte polynomial for the signed graph corresponding to $M$ in this case is (using its 55
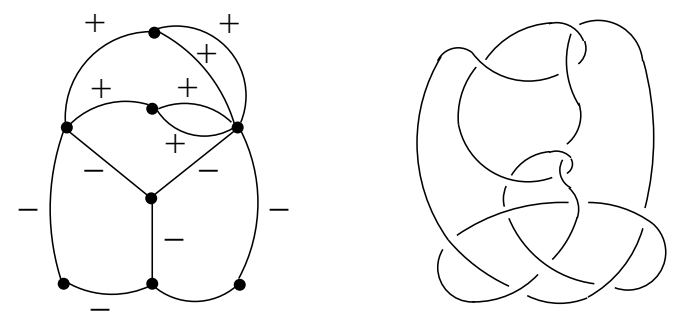

Figure 11. A knot obtained from positive tensor product using $9_{49}$ and $4_{1}$.

spanning trees):

$$
\begin{aligned}
& B_{+}^{2}\left(2 x_{-}^{2} A_{-}^{3} y_{-} B_{-}+3 x_{-}^{3} A_{-}^{2} B_{-}^{2}+x_{-}^{5} B_{-}^{2}+2 x_{-}^{4} A_{-} B_{-}^{2}\right. \\
+ & \left.2 x_{-} A_{-}^{4} y_{-} B_{-}+2 x_{-}^{2} A_{-}^{3} B_{-}^{2}+x_{-} A_{-}^{4} B_{-}^{2}+A_{-}^{5} y_{-}^{2}+A_{-}^{5} y_{-} B_{-}\right) \\
+ & \left(A_{+} y_{+}+A_{+} B_{+}\right)\left(2 x_{-}^{3} A_{-} B_{-}^{3}+3 x_{-}^{2} A_{-}^{2} B_{-}^{3}+4 x_{-} A_{-}^{3} y_{-} B_{-}^{2}\right. \\
+ & x_{-} A_{-}^{3} B_{-}^{3}+2 A_{-}^{4} y_{-}^{2} B_{-}+A_{-}^{4} y_{-} B_{-}^{2}+x_{-}^{4} B_{-}^{3} \\
+ & \left.x_{-}^{3} A_{-} y_{-} B_{-}^{2}+2 x_{-}^{2} A_{-}^{2} y_{-} B_{-}^{2}+A_{-}^{4} y_{-}^{3}+2 x_{-} A_{-}^{3} y_{-}^{2} B_{-}\right) .
\end{aligned}
$$

To verify that this is correct, we calculate the bracket polynomial from it using Theorem 6.1. This gives us

$$
-A^{19}+2 A^{15}-4 A^{11}+4 A^{7}-5 A^{3}+4 A^{-1}-3 A^{-5}+2 A^{-9} .
$$

Since the writhe of this projection of $9_{49}$ is 9 , we obtain the Jones polynomial of $9_{49}$ :

$$
\begin{aligned}
V\left(9_{49}\right) & =\left(-A^{-3}\right)^{w\left(9_{49}\right)}\left\langle 9_{49}\right\rangle \\
& =-A^{-27}\left(-A^{19}+2 A^{15}-4 A^{11}+4 A^{7}-5 A^{3}+4 A^{-1}-3 A^{-5}+2 A^{-9}\right) \\
& =A^{-8}-2 A^{-12}+4 A^{-16}-4 A^{-20}+5 A^{-24}-4 A^{-28}+3 A^{-32}-2 A^{-36} \\
& =t^{2}-2 t^{3}+4 t^{4}-4 t^{5}+5 t^{6}-4 t^{7}+3 t^{8}-2 t^{9} .
\end{aligned}
$$


This matches the known Jones polynomial for $9_{49}$.

We next find the polynomials $T(N \backslash e), T(N / e), T_{L}(N, e)$ and $T_{C}(N, e)$. We calculate the first two by drawing the spanning trees of $N \backslash e$ and $N / e$, then the latter two are obtained from the first two by applying the exception rules. We have

$$
\begin{aligned}
T(N \backslash e) & =x_{+}^{2} B_{+}+x_{+} y_{+} A_{+}, \\
T(N / e) & =x_{+} B_{+}^{2}+y_{+} A_{+} B_{+}+y_{+}^{2} A_{+}, \\
T_{L}(N, e) & =A_{+}^{2} B_{+}+A_{+}^{2} y_{+}, \\
T_{C}(N, e) & =x_{+} B_{+}^{2}+A_{+} B_{+}^{2}+y_{+} A_{+} B_{+} .
\end{aligned}
$$

We may now replace $B_{+}, y_{+}$and $A_{+}$in $T(M)$ with $T_{C}(N, e), T(N / e)$, and $T_{L}(N, e)$ (since $x_{+}$does not appear in $T(M))$ respectively to obtain $T\left(M \otimes_{+} N\right)$ :

$$
\begin{aligned}
T\left(M \otimes_{+} N\right) & =\left(x_{+} B_{+}^{2}+A_{+} B_{+}^{2}+y_{+} A_{+} B_{+}\right)^{2}\left(2 x_{-}^{2} A_{-}^{3} y_{-} B_{-}\right. \\
& +3 x_{-}^{3} A_{-}^{2} B_{-}^{2}+x_{-}^{5} B_{-}^{2}+2 x_{-}^{4} A_{-} B_{-}^{2}+2 x_{-} A_{-}^{4} y_{-} B_{-} \\
& \left.+2 x_{-}^{2} A_{-}^{3} B_{-}^{2}+x_{-} A_{-}^{4} B_{-}^{2}+A_{-}^{5} y_{-}^{2}+A_{-}^{5} y_{-} B_{-}\right) \\
& +\left[\left(A_{+}^{2} B_{+}+A_{+}^{2} y_{+}\right)\left(x_{+} B_{+}^{2}+y_{+} A_{+} B_{+}+y_{+}^{2} A_{+}\right)\right. \\
& \left.+\left(A_{+}^{2} B_{+}+A_{+}^{2} y_{+}\right)\left(x_{+} B_{+}^{2}+A_{+} B_{+}^{2}+y_{+} A_{+} B_{+}\right)\right]\left(2 x_{-}^{3} A_{-} B_{-}^{3}\right. \\
& +3 x_{-}^{2} A_{-}^{2} B_{-}^{3}+4 x_{-} A_{-}^{3} y_{-} B_{-}^{2}+x_{-} A_{-}^{3} B_{-}^{3}+2 A_{-}^{4} y_{-}^{2} B_{-} \\
& +A_{-}^{4} y_{-} B_{-}^{2}+x_{-}^{4} B_{-}^{3}+x_{-}^{3} A_{-} y_{-} B_{-}^{2}+2 x_{-}^{2} A_{-}^{2} y_{-} B_{-}^{2} \\
& \left.+A_{-}^{4} y_{-}^{3}+2 x_{-} A_{-}^{3} y_{-}^{2} B_{-}\right) .
\end{aligned}
$$

We can then calculate the bracket polynomial and the Jones polynomial for $K_{M \otimes_{+} N}$ :

$$
\begin{aligned}
\left\langle K_{M \otimes_{+} N}\right\rangle & =-A^{27}+4 A^{23}-9 A^{19}+14 A^{15}-17 A^{11}+19 A^{7} \\
& -18 A^{3}+13 A^{-1}-9 A^{-5}+3 A^{-9}-A^{-17}+A^{-21} .
\end{aligned}
$$

The writhe of the knot projection diagram corresponding to $M \otimes_{+} N$ is 1 . Hence

$$
\begin{aligned}
& V_{K_{M \otimes_{+} N}}(t)=\left(-A^{-3}\right)^{w\left(K_{M \otimes_{+} N}\right)}\left\langle K_{M \otimes_{+} N}\right\rangle \\
= & A^{24}-4 A^{20}+9 A^{16}-14 A^{12}+17 A^{8}-19 A^{4}+18-13 A^{-4}+9 A^{-8}-3 A^{-12}+A^{-20}-A^{-24} \\
= & t^{-6}-4 t^{-5}+9 t^{-4}-14 t^{-3}+17 t^{-2}-19 t^{-1}+18-13 t+9 t^{2}-3 t^{3}+t^{5}-t^{6} .
\end{aligned}
$$

Using the DT code $[-4,-22,-8,-20,14,24,18,26,10,-6,-2,12,16]$ obtained from the diagram in Figure 11, we get the same Jones polynomial using Knotscape [5].

Example 6.3. Our next example deals with a knot family with a parameter $k$. We will illustrate the details using the case of $k=3$ (illustrated in Figure 12) and then show our computation results for the cases of $k=5,7$ and 9 . Notice that the projection diagram $D$ is non-alternating. For $k=3$, there are 19 crossings in the diagram, 10 positive and 9 negative with respect to the shaded regions. The corresponding signed graph $G$ of this diagram (using the shaded regions in the figure as the vertices) is shown on the right side. A labelling of the edges of $G$ is given in the figure as well.

Denote the edge labelled by 19 in the figure by $g$. Let $G \backslash g$ be the graph obtained from $G$ by deleting $g$ and let $G / g$ be the graph obtained from $G$ by contracting $g$, that is, the vertices incident to $g$ are identified as one single vertex after $g$ is removed. See Figure 13, By the recursive formula (6), we have

$$
T(G)=B_{+} T(G \backslash g)+A_{+} T(G / g) .
$$

Thus we will concentrate on computing $T(G \backslash g)$ and $T(G / g)$. Notice that $G \backslash g$ and $G / g$ can be obtained from the simple graphs $M_{1}$ and $M_{2}$ shown in Figure14 by applying repeated tensor product operations: 

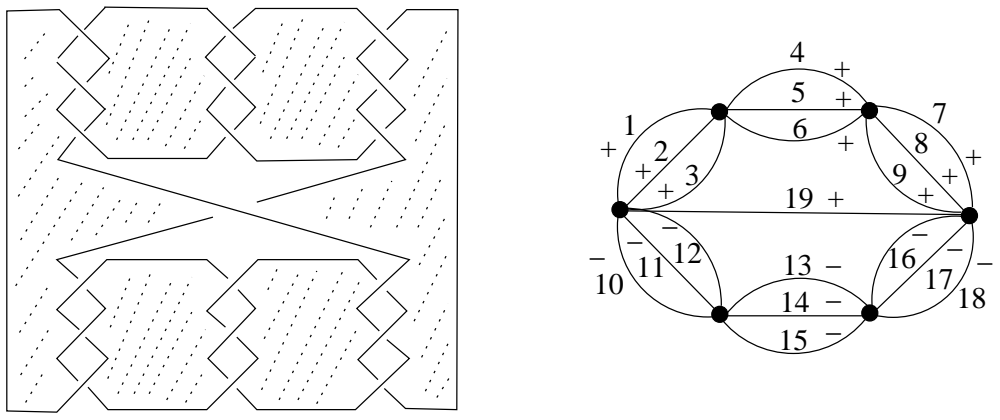

FiguRE 12. A 19 crossing knot diagram and its corresponding signed graph $G$.

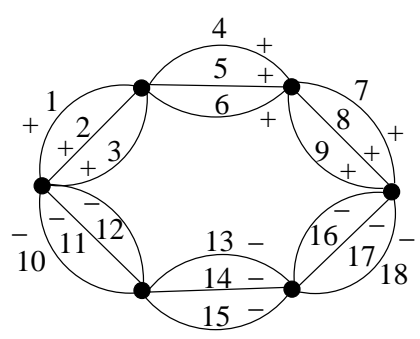

Gle

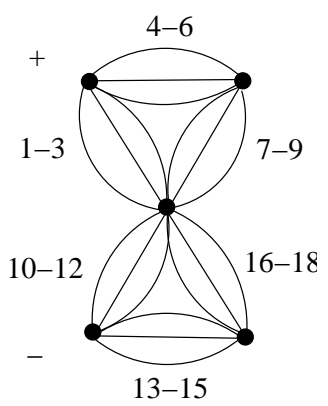

G/e

FigurE 13. The graphs obtained from the graph $G$ in Figure 12 by deletion and contraction.

$G \backslash g=\left(\left(\left(\left(M_{1} \otimes_{+} S_{+}\right) \otimes_{-} S_{-}\right) \otimes_{+} T_{+}\right) \otimes_{-} T_{-}\right.$and $G / g=\left(\left(\left(M_{2} \otimes_{+} S_{+}\right) \otimes_{-} S_{-}\right) \otimes_{+} T_{+}\right) \otimes_{-} T_{-}$, where $S_{-}, S_{+}, T_{-}$and $T_{+}$are shown in Figure 15 for the case of $k=3$. Notice that the identifying edge $e$ is marked in the figure.

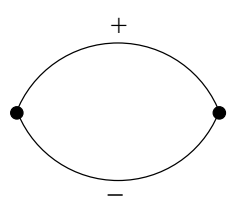

$\mathrm{M}_{1}$

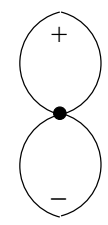

$\mathrm{M}_{2}$

Figure 14. Two very simple graphs.

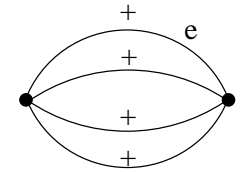

$\mathrm{T}_{+}$

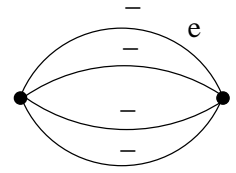

$\mathrm{T}_{-}$

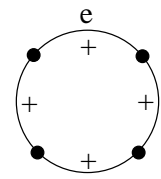

$\mathrm{S}_{+}$

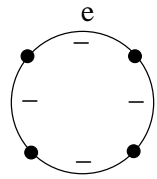

$\mathrm{S}-$

FIGURE 15. These graphs are also called stretching and thickening graphs.

Since $T\left(M_{1}\right)=x_{+} B_{-}+y_{+} A_{-}, T\left(M_{2}\right)=y_{+} y_{-}$and each of $S_{-}, S_{+}, T_{-}$and $T_{+}$uses only one sign, $T(G \backslash g)$ and $T(G / g)$ can be computed by making the following substitutions twice starting from 
$x_{+} B_{-}+y_{+} A_{-}$and $y_{+} y_{-}$. (We leave it as an exercise to our reader to compute the polynomials $T\left(S_{ \pm} \backslash e\right), T\left(S_{ \pm} / e\right), T\left(T_{ \pm} \backslash e\right), T\left(T_{ \pm} / e\right), T_{C}\left(S_{ \pm}, e\right), T_{C}\left(T_{ \pm}, e\right), T_{L}\left(S_{ \pm}, e\right)$ and $\left.T_{L}\left(T_{ \pm}, e\right).\right)$

$$
\begin{aligned}
A_{-} & \mapsto A_{-}\left(y_{-}^{2}+y_{-} B_{-}+B_{-}^{2}\right), \quad B_{-} \mapsto B_{-}^{3}, \\
A_{+} & \mapsto A_{+}\left(y_{+}^{2}+y_{+} B_{+}+B_{+}^{2}\right), \quad B_{+} \mapsto B_{+}^{3}, \\
x_{-} & \mapsto B_{-}^{2} x_{-}+\left(y_{-}+B_{-}\right) A_{-} y_{-}, \quad y_{-} \mapsto y_{-}^{3}, \\
x_{+} & \mapsto B_{+}^{2} x_{+}+\left(y_{+}+B_{+}\right) A_{+} y_{+}, \quad y_{+} \mapsto y_{+}^{3} .
\end{aligned}
$$

After making the substitutions listed in Theorem 6.1 and substituting $t^{-\frac{1}{4}}$ for $A$ in Equation (13) (with $w(K)=-1$ ), we obtain

$$
\begin{aligned}
V_{K}(t) & =t^{-10}\left(1-4 t+12 t^{2}-26 t^{3}+49 t^{4}-74 t^{5}+96 t^{6}-112 t^{7}+110 t^{8}\right. \\
& \left.-97 t^{9}+77 t^{10}-47 t^{11}+23 t^{12}-8 t^{13}-2 t^{14}+3 t^{15}-t^{16}+t^{17}\right) .
\end{aligned}
$$

This matches the computation result obtained by using Knotscape [5]. Use the same approach, we can compute the Jones polynomials for larger values of $k$. The result for $k=5,7$ and 9 are listed below. The computation time for the case of $k=9$ is about 10 minutes using Maple on a PC.

For $k=5$,

$$
\begin{aligned}
V_{K}(t) & =t^{-26}\left(1-6 t+26 t^{2}-91 t^{3}+275 t^{4}-737 t^{5}+1796 t^{6}-4021 t^{7}+8366 t^{8}-16284 t^{9}\right. \\
& +29818 t^{10}-51606 t^{11}+84676 t^{12}-132106 t^{13}+196368 t^{14}-278544 t^{15}+377546 t^{16} \\
& -489336 t^{17}+606846 t^{18}-720177 t^{19}+817720 t^{20}-887911 t^{21}+920952 t^{22}-911068 t^{23} \\
& +857489 t^{24}-765053 t^{25}+643579 t^{26}-505933 t^{27}+366267 t^{28}-237242 t^{29}+128459 t^{30} \\
& -45354 t^{31}-11121 t^{32}+43431 t^{33}-56574 t^{34}+56418 t^{35}-48576 t^{36}+37646 t^{37} \\
& -26696 t^{38}+17478 t^{39}-10594 t^{40}+5941 t^{41}-3081 t^{42}+1466 t^{43}-637 t^{44} \\
& \left.+250 t^{45}-86 t^{46}+26 t^{47}-6 t^{48}+t^{49}\right) .
\end{aligned}
$$

For $k=7$,

$$
\begin{aligned}
V_{K}(t) & =t^{-50}\left(1-8 t+43 t^{2}-183 t^{3}+666 t^{4}-2157 t^{5}+6370 t^{6}-17425 t^{7}+44654 t^{8}-108067 t^{9}\right. \\
& +248536 t^{10}-545847 t^{11}+1149387 t^{12}-2328122 t^{13}+4548764 t^{14}-8593271 t^{15} \\
& +15728483 t^{16}-27941544 t^{17}+48253003 t^{18}-81115378 t^{19}+132896097 t^{20}-212430488 t^{21} \\
& +331612373 t^{22}-505966329 t^{23}+755122019 t^{24}-1103084529 t^{25}+1578177868 t^{26} \\
& -2212528476 t^{27}+3040964638 t^{28}-4099238067 t^{29}+5421525110 t^{30}-7037249318 t^{31} \\
& +8967357925 t^{32}-11220302612 t^{33}+13788073932 t^{34}-16642729060 t^{35}+19733901580 t^{36} \\
& -22987768175 t^{37}+26307888783 t^{38}-29578176531 t^{39}+32668072879 t^{40}-35439739995 t^{41} \\
& +37756834115 t^{42}-39494183464 t^{43}+40547500331 t^{44}-40842187270 t^{45}+40340281916 t^{46} \\
& -39044758086 t^{47}+37000613964 t^{48}-34292527561 t^{49}+31039223375 t^{50}-27385045791 t^{51} \\
& +23489568932 t^{52}-19516242140 t^{53}+15621197678 t^{54}-11943255900 t^{55}+8596013866 t^{56} \\
& -5662618702 t^{57}+3193491734 t^{58}-1206952613 t^{59}-307642091 t^{60}+1384824407 t^{61} \\
& -2076750500 t^{62}+2446499548 t^{63}-2561637408 t^{64}+2488610216 t^{65}-2288339306 t^{66} \\
& +2013210200 t^{67}-1705443587 t^{68}+1396707072 t^{69}-1108711902 t^{70}+854497057 t^{71} \\
& -640099923 t^{72}+466343256 t^{73}-330533561 t^{74}+227925671 t^{75}-152882699 t^{76} \\
& +99711533 t^{77}-63198913 t^{78}+38898252 t^{79}-23227709 t^{80}+13441772 t^{81}-7528452 t^{82}
\end{aligned}
$$




$$
\begin{aligned}
& +\quad 4074514 t^{83}-2126998 t^{84}+1068635 t^{85}-515390 t^{86}+237854 t^{87}-104637 t^{88}+43667 t^{89} \\
& \left.-17180 t^{90}+6321 t^{91}-2150 t^{92}+666 t^{93}-183 t^{94}+43 t^{95}-8 t^{96}+t^{97}\right) .
\end{aligned}
$$

For $k=9$, the polynomial is too large to list, so we only list a few terms below:

$$
\begin{aligned}
V_{K}(t)= & t^{-82}\left(1-10 t+64 t^{2}-319 t^{3}+1345 t^{4}-5008 t^{5}+\cdots\right. \\
& +\cdots-20193935024459 t^{97}-101497138129454 t^{98}+\cdots \\
& \left.\cdots-5008 t^{156}+1345 t^{157}-319 t^{158}+64 t^{159}-10 t^{160}+t^{161}\right) .
\end{aligned}
$$

The knots constructed this way are non-alternating since the Jones polynomials are not alternating. It follows that the crossing numbers of these knots (at least for the ones we have computed above) are at least $2 k^{2}$ since the breadth of the polynomials is $2 k^{2}-1$ and the crossing number of a non-alternating knot is strictly larger than the breadth of its Jones polynomial.

The computation of the above example with $k=9$ took only minutes on a PC using Maple. However, it is not always possible to compute the Tutte polynomial quickly through the variable substitutions given in the last section. It would be interesting to see what (non-alternating) knots can be constructed using graph tensor product whose Jones polynomials (or the breadths of their Jones polynomials) can be computed with polynomial runtime. This is a possible direction of future research. The authors also intend to investigate the possibilities of extending their results to other knot polynomials or other graph invariants that are related to the Tutte polynomial.

\section{ACKNOWLEDGEMENT}

We wish to thank Professor T. G. Lucas for providing the main idea behind Lemma 2.7.

\section{REFERENCES}

[1] B. Bollobás and O. Riordan, A Tutte Polynomial for Coloured Graphs, Combinatorics, Probability and Computing 8 (1999), 45-93.

[2] W. Bruns, U. Wetter, Determinantal Rings, Springer Lecture Notes in Mathematics \# 1327, Springer-Verlag BerlinHeidelberg 1988.

[3] T. Brylawski, The Tutte Polynomial I: General theory, in: Matroid Theory and its Applications, ed. A. Barlotti, Liguori Editore, S.r.I, 1982, 125-275.

[4] Brylawski and Oxley, The Tutte Polynomial and its Applications, Matroid Applications (ed. N. White), Cambridge University Press (1992), 123-225.

[5] J. Hoste and M. Thistlethwaite, Knotscape, http://www.math.utk. edu/ morwen/knotscape.html.

[6] S. Huggett, On tangles and matroids, Journal of Knot Theory and its Ramifications 14 (2005), 919-929.

[7] F. Jaeger, D.L. Vertigan and D.J.A. Welsh, On the Computational Complexity of the Jones and Tutte Polynomials, Mathematical Proceedings of the Cambridge Philosophical Society 108 (1990), 35-53.

[8] L.H. Kauffman, New Invariants in the Theory of Knots, American Mathematical Monthly 95(3) (1988), 195-242.

[9] L.H. Kauffman, A Tutte Polynomial for Signed Graphs, Discrete Applied Mathematics 25 (1989), $105-127$.

[10] K. Murasugi, Jones Polynomials and Classical Conjectures in Knot Theory, Topology 26(2) (1987), $187-194$.

[11] M. B. Thistlethwaite, A Spanning Tree Expansion for the Jones Polynomial, Topology 26(3) (1987), 297-309.

[12] W.T. Tutte, A contribution to the Theory of Chromatic polynomials, Canadian Journal of Mathematics 6 (1954), $80-91$.

Department of Mathematics and Statistics, UnC Charlotte, Charlotte, nC 28223

E-mail address: ydiao@uncc.edu, ghetyei@uncc.edu, kehinson@uncc.edu 